TRANSACTIONS OF THE

AMERICAN MATHEMATICAL SOCIETY

Volume 356, Number 1, Pages 319-344

S 0002-9947(03)03336-

Article electronically published on August 25, 2003

\title{
EIGENVALUE AND GAP ESTIMATES FOR THE LAPLACIAN ACTING ON $p$-FORMS
}

\author{
PIERRE GUERINI AND ALESSANDRO SAVO
}

\begin{abstract}
We study the gap of the first eigenvalue of the Hodge Laplacian acting on $p$-differential forms of a manifold with boundary, for consecutive values of the degree $p$.

We first show that the gap may assume any sign. Then we give sufficient conditions on the intrinsic and extrinsic geometry to control it. Finally, we estimate the first Hodge eigenvalue of manifolds whose boundaries have some degree of convexity.
\end{abstract}

Let $\left(M^{n}, g\right)$ be an $n$-dimensional compact, oriented Riemannian manifold with (possibly empty) smooth boundary $\partial M^{n}$. For all $p \in\{0, \ldots, n\}$ we consider the Laplacian $\Delta_{p}$ (often denoted simply by $\Delta$ ) which acts on smooth differential $p$-forms $\omega \in \Lambda^{p}(M)$ by

$$
\Delta_{p} \omega:=(d \delta+\delta d) \omega
$$

where $d$ is the exterior derivative and $\delta:=(-1)^{n(p+1)+1} * d *$ is the codifferential, * being the Hodge operator. $\Delta_{p}$ is often called the Hodge (or Hodge-de Rham) Laplacian of $M$.

If $\partial M \neq \emptyset$, we consider the following eigenvalue problem defined by the absolute boundary conditions:

$$
\left\{\begin{array}{l}
\Delta \omega=\mu \omega, \\
J^{\star} i_{\tilde{\nu}} \omega=J^{\star} i_{\tilde{\nu}} d \omega=0,
\end{array}\right.
$$

where $J$ is the inclusion $\partial M \hookrightarrow M$ and $\tilde{\nu}$ is the inward unit normal vector at each point of $\partial M$.

This is a generalization of the Neumann boundary problem for functions. We say that the form $\omega$ is tangential on $\partial M$ if $J^{\star} i_{\tilde{\nu}} \omega=0$, that is, if $\omega$ vanishes whenever one of its arguments is a vector normal to the boundary. Hence $\omega$ satisfies the absolute boundary conditions if and only if both $\omega$ and $d \omega$ are tangential.

The other usual boundary problem for differential forms is given by the relative boundary conditions: $J^{\star} \omega=J^{\star} \delta \omega=0$. It generalizes the Dirichlet boundary problem for functions. Note that the Hodge operator exchanges the two boundary conditions. We use the notation

$\mu_{1, p}(M):=$ first positive eigenvalue of $\Delta_{p}$ if $\left\{\begin{array}{l}\partial M=\emptyset, \text { or } \\ \partial M \neq \emptyset \text { for the absolute conditions }\end{array}\right.$

Received by the editors January 13, 2003.

2000 Mathematics Subject Classification. Primary 58J50; Secondary 58J32.

Key words and phrases. Hodge Laplacian, eigenvalues, gaps, convex manifolds. 
and, if $\partial M \neq \emptyset$,

$$
\lambda_{1, p}(M):=\text { first positive eigenvalue of } \Delta_{p} \text { for the relative conditions. }
$$

Note that $\mu_{1,0}(M)$ (resp. $\left.\lambda_{1,0}(M)\right)$ is just the first nonzero eigenvalue of the Laplacian on functions for the Neumann (resp. Dirichlet) conditions.

Let us describe the scopes of the paper.

In the first two sections we compare the Hodge eigenvalues for consecutive values of the degree $p$; namely, we study what we call the $p$-gap of the manifold,

$$
\mu_{1, p}(M)-\mu_{1, p-1}(M) .
$$

In the third section, we give a lower bound for the first Hodge eigenvalue of a Riemannian manifold with a boundary having a suitable degree of convexity.

Now the 1-gap is always nonpositive, because it always turns out that $\mu_{1,1}(M) \leq$ $\mu_{1,0}(M)$. In [16] and 17] Takahashi shows that any closed manifold $M$ admits two metrics: one with $\mu_{1,1}<\mu_{1,0}$ and the other with $\mu_{1,1}=\mu_{1,0}$. Hence, the 1-gap does not impose any restrictions on the topology of the underlying manifold. We show in Section 1 that the same is true for manifolds with boundary (see Theorem 1.1); more generally we show that the sign of the $p$-gap (at least if $p \leq n-2$ ) has nothing to do with topology. We remark that our construction holds also for Euclidean metrics: in each diffeomorphism class, we can find three Euclidean domains for which the $p$-gap is positive, negative and zero (if $p=2, \ldots, n-2$ ), respectively, and a pair of Euclidean domains for which $\mu_{1,1}<\mu_{1,0}$ and $\mu_{1,1}=$ $\mu_{1,0}$, respectively. This is not a priori obvious, because Euclidean domains have often special properties: for example they always satisfy the well-known inequality $\mu_{1,0}(M)<\mu_{1, n}(M)=\lambda_{1,0}(M)$ (see [4] and the references there). This does not hold for general Riemannian manifolds with boundary (the excision of a small ball from a Euclidean sphere, for instance, leads to a small eigenvalue for the Dirichlet problem on the resulting manifold, but not for the Neumann problem).

Having said that, it would be interesting to see what geometric conditions guarantee that the $p$-gap has a definite sign. Loosely speaking, we show in section 2 that convexity is one of these conditions.

More precisely, we prove (Theorem 2.6) that under the assumption of " $p$-convexity", defined below, a Euclidean domain satisfies $\mu_{1, p}(M) \geq \mu_{1, p-1}(M)$. In particular, a convex Euclidean domain has nonnegative $p$-gap for all $p$, and therefore the sequence $\left\{\mu_{1, p}(M)\right\}_{p=0, \ldots, n}$ is nondecreasing in that case. Note that by what we said above, this fact does not hold in general. More generally, in section 2 we give lower bounds of the $p$-gap of an isometric immersion of our Riemannian manifold (with or without boundary) in some Euclidean space and we apply this result to show that the $p$-gap of a convex spherical domain is not less than that of a hemisphere (Theorem 2.8). Consequently the (relative or absolute) Hodge spectrum of convex Euclidean and spherical domains is always bounded below by the first Neumann eigenvalue on functions. We also give an extrinsic sufficient condition for having $\mu_{1,1}=\mu_{1,0}$ on an immersed submanifold of Euclidean space (Corollary 2.4).

All the above facts follow from a lower bound for the $L^{2}$-norm of the energy of co-closed forms on the immersed manifold (Theorem 2.1) which should be regarded as the main result of Section 2. In particular, when applied to the volume form of $M$, Theorem 2.1 gives back an estimate of Reilly on the eigenvalue $\mu_{1,0}$; see [14] and Remark 2.2 below. 
Finally, in Section [3, we give in Theorem 3.3 a lower bound of the first Hodge eigenvalues for arbitrary Riemannian manifolds with boundary in terms of the principal curvatures (more generally, what we call the $p$-curvatures) of the boundary, and the curvature operator of the manifold. The estimate comes from a general lower bound on the ratio between the integral of a nonnegative function on $\partial M$ and $M$, respectively, which is of independent interest (see Theorem 3.1).

We find in particular that convex hyperbolic domains whose principal curvatures are bounded below by 1 (all geodesic balls belong to this family) have their relative Hodge eigenvalues $\lambda_{1, p}(M)$ bounded below by a positive constant independent of the inner radius, provided that their dimension is sufficiently large with respect to the degree $p$ (see Corollary 3.5). This inequality somewhat generalizes a well-known property of the first Dirichlet eigenvalue of hyperbolic domains (convex or not).

In the first three sections we state the main results in detail, leaving the proofs of the main theorems and additional remarks to the last section of the paper.

Definitions and basic facts. We first recall some basic facts and notations which we will be used in the sequel. As the Hodge Laplacian commutes with both $d$ and $\delta$, it preserves closed (resp. co-closed) $p$-forms; we denote by $\mu_{1, p}^{\prime}(M)\left(\operatorname{resp} . \mu_{1, p}^{\prime \prime}(M)\right)$ the smallest positive eigenvalue corresponding to closed (resp. co-closed) forms. The Hodge decomposition theorem implies that

$$
\mu_{1, p}(M)=\min \left\{\mu_{1, p}^{\prime}(M), \mu_{1, p}^{\prime \prime}(M)\right\}
$$

and

$$
\mu_{1, p}^{\prime \prime}(M)=\mu_{1, p+1}^{\prime}(M)
$$

for $p \geq 0$. This carries through if $M$ has a non-empty boundary, for both the absolute and relative conditions. In particular, one always has $\mu_{1,1} \leq \mu_{1,0}$ and the equality holds iff $\mu_{1,1}^{\prime \prime} \geq \mu_{1,1}^{\prime}$.

We also recall that, as the Hodge operator is an isometry commuting with the Laplacian, one has, for all $p=0, \ldots, n$ :

$$
\mu_{1, p}(M)=\mu_{1, n-p}(M) \text { if } \partial M=\emptyset \text { and } \mu_{1, p}(M)=\lambda_{1, n-p}(M) \text { if } \partial M \neq \emptyset .
$$

We adopt the following notations:

$$
\begin{aligned}
(n-1) K & :=\text { lower bound of the Ricci curvature of } M, \\
\gamma & :=\text { lower bound of the eigenvalues of the curvature operator of } M .
\end{aligned}
$$

Let $\omega$ be a $p$-form. The Bochner (or Bochner-Weitzenböck) formula is the identity valid at any point of $M$ :

$$
\langle\Delta \omega, \omega\rangle=\|\nabla \omega\|^{2}+\frac{1}{2} \Delta\left(\|\omega\|^{2}\right)+\left\langle W_{p}(\omega), \omega\right\rangle,
$$

where $\langle\cdot, \cdot\rangle$ is the pointwise inner product of forms, and where $W_{p}$ is a selfadjoint endomorphism of $\Lambda^{p}(M)$, called the curvature term. Then $W_{1}=$ Ric and, by [5]:

$$
W_{p} \geq p(n-p) \gamma \cdot I d
$$

Now fix $x \in \partial M$ and $p=1, \ldots, n-1$. Let

$$
\begin{aligned}
\sigma_{p}(x) & :=\operatorname{sum} \text { of the lowest } p \text { principal curvatures of } \partial M \text { at } x, \text { and } \\
\sigma_{p} & :=\inf _{\partial M} \sigma_{p}(x) .
\end{aligned}
$$


In other words, $\sigma_{p}$ is the lower bound, over $\partial M$, of all its $p$-curvatures (sums of any set of $p$ principal curvatures). In particular:

$$
\begin{aligned}
\sigma_{1} & =\text { lower bound of the principal curvatures of } \partial M, \\
\frac{\sigma_{n-1}}{n-1} & =H=\text { lower bound of the mean curvature of } \partial M .
\end{aligned}
$$

One sees that $H \geq \sigma_{p} / p \geq \sigma_{1}$ for all $p$.

We will say that $\partial M$ is $p$-convex if $\sigma_{p} \geq 0$. Hence the condition of $p$-convexity is weaker than the usual convexity and stronger than the $(n-1)$-convexity (i.e. nonnegative mean curvature).

\section{General Results on the $p$-GaP}

We prove the following fact.

Theorem 1.1. Let $M$ be a smooth compact manifold with boundary of dimension $n \geq 3$ and let $p$ be an integer in $\{0, \ldots, n-1\}$. Then there exist metrics $g_{p}, g_{p}^{\prime}, g_{p}^{\prime \prime}$ on $M$ such that:

(1) If $p \neq n-2, n-1$, then $\mu_{1, p}\left(M, g_{p}\right)>\mu_{1, p+1}\left(M, g_{p}\right)$.

(2) If $p \neq 0$, then $\mu_{1, p}\left(M, g_{p}^{\prime}\right)<\mu_{1, p+1}\left(M, g_{p}^{\prime}\right)$.

(3) If $p \neq n-1$, then $\mu_{1, p}\left(M, g_{p}^{\prime \prime}\right)=\mu_{1, p+1}\left(M, g_{p}^{\prime \prime}\right)$.

If $M$ is a Euclidean (resp. spherical) domain, then the metrics above can be chosen to be Euclidean (resp. spherical).

The proof is given in Section 4.1] and uses a result proved in [9] (see Theorem 4.1)

As shown in Section [2, the sequence $\mu_{1, p}$ is nondecreasing in $p$ when $M$ is a convex Euclidean domain. Hence, Theorem 1.1 gives counterexamples to this fact in the general case.

In Theorem 1.1 of 9 , it is shown that one can prescribe any finite part of the $p$-spectrum for $p$ in a suitable range. This means that many cases in Theorem 1.1 are direct consequences of 9 . However, Assertion 1 for $p=0,1$, Assertion 2 for $p=1, n-1$ and Assertion 3 for $p=0,1$ do not follow from [9], and we must give an independent derivation of them here.

A short comment about the missing cases in Theorem 1.1 above. Assertion 1 (resp. 2) is never satisfied when $p=n-1$ (resp. $p=0$ ). But Assertion 1 is an open problem if $p=n-2$; similarly, Assertion 3 is open if $p=n-1$. Our method to control the gaps between the eigenvalues is to make one eigenvalue tend to zero while the others remain bounded below. Now the Faber-Frahn inequality makes it impossible to get on a Euclidean domain a small eigenvalue $\mu_{1, n-1}$ without getting at the same time a small eigenvalue $\mu_{1, n-2}$.

In a similar spirit, Takahashi shows in [17] that, given a closed manifold $M$ of dimension $n \geq 4$ and an integer $p=2, \ldots, n-2$, there exist three metrics $g_{1}, g_{2}, g_{3}$ on $M$ such that his "gap" $\mu_{1, p}\left(M, g_{i}\right)-\mu_{1,0}\left(M, g_{i}\right)$ is positive, negative and zero, respectively. As an immediate by-product of our proof of Theorem 1.1] we prove part of Takahashi's result for manifolds with boundary and in particular for Euclidean or spherical domains, that is, when the inner metric is prescribed (see Corollary 4.4).

The results of this section show that much liberty remains in the construction of gaps, even if we impose a strong rigidity on the metric. To get rigidity for the gaps, 
one must assume additional conditions, for example, convexity of the boundary. We will address this problem in the next section.

\section{Gap of isometric immersions}

In this section we consider an isometric immersion of the Riemannian manifold $M^{n}$ into some Euclidean space $\mathbf{R}^{d}$.

If $\nu$ is a vector normal to $M$, we consider the shape operator $S_{\nu}$ relative to $\nu$; it is defined by the identity $\left\langle S_{\nu}(X), Y\right\rangle=\langle L(X, Y), \nu\rangle$ for all $X, Y \in T M$, where $L$ is the second fundamental form of the immersion. We extend $S_{\nu}$, by derivation, to a selfadjoint endomorphism of $\Lambda^{p}(M)$, denoted by $S_{\nu, p}$ and given by the well-known formula:

$$
S_{\nu, p}(\omega)\left(X_{1}, \ldots, X_{p}\right)=\sum_{i=1}^{p} \omega\left(X_{1}, \ldots, S_{\nu}\left(X_{i}\right), \ldots, X_{p}\right) .
$$

If $\left(\nu_{1}, \ldots, \nu_{m}\right)$ is an orthonormal basis of the normal bundle of $M$ at any fixed point (so that $m$ is the codimension of the immersion), then the formula

$$
T_{p}=\sum_{\alpha=1}^{m}\left(S_{\nu_{\alpha}, p}\right)^{2}
$$

defines a selfadjoint, nonnegative endomorphism $T_{p}$ of $\Lambda^{p}(M)$ which does not depend on the orthonormal basis chosen. In particular, for any $p$-form $\omega$ :

$$
\left\langle T_{p}(\omega), \omega\right\rangle=\sum_{\alpha=1}^{m}\left\|S_{\nu_{\alpha}, p}(\omega)\right\|^{2} .
$$

The main result of this section is a general lower bound for the integral energy of a co-closed $p$-form on $M$.

Theorem 2.1. Let $M^{n} \rightarrow \mathbf{R}^{d}$ be an isometric immersion and $\omega$ a co-closed $p$-form on $M$, with $p=1, \ldots, n$. If $\partial M \neq \emptyset$, we assume in addition that $\omega$ is tangential on $\partial M$, that is, it vanishes whenever one of its arguments is a vector normal to the boundary. Then

$$
\int_{M}\left\{\|\nabla \omega\|^{2}+\left\langle T_{p}(\omega), \omega\right\rangle+(p-1)\|d \omega\|^{2}\right\} \geq p \mu_{1, p}^{\prime}(M) \int_{M}\|\omega\|^{2} .
$$

The inequality is sharp for any eigenform associated to $\mu_{1, p}^{\prime \prime}\left(\mathbf{S}^{n}\right)$, where $\mathbf{S}^{n} \rightarrow$ $\mathbf{R}^{n+1}$ is the standard immersion of the canonical sphere.

For the proof, see Section 4.2 .

Remark 2.2. When applied to the volume form $d v o l$ of $M^{n}$ (now assumed without boundary), Theorem 2.1 reduces to the following well-known estimate, due to Reilly [14], for the first eigenvalue of the Laplacian on functions:

$$
\mu_{1,0}(M) \leq n \cdot \frac{\int_{M}\|\eta\|^{2}}{\operatorname{vol}(M)} .
$$

where $\eta$ is the mean curvature vector of the immersion.

In fact, $d v o l$ is parallel; by Hodge duality $\mu_{1, n}^{\prime}(M)=\mu_{1,0}(M)$, and the identity $\left\langle T_{n}(d v o l), d v o l\right\rangle=n^{2}\|\eta\|^{2}$ is immediate from the definitions. 
For $p=1$ one also gets a lower bound for the energy of vector fields, which are divergence free and tangent to the boundary, by the first Neumann eigenvalue of the manifold.

We then deduce from Theorem 2.1 a lower bound for the gap $\mu_{1, p}^{\prime \prime}(M)-\mu_{1, p}^{\prime}(M)$ in terms of the curvature term $W_{p}$ of Bochner formula (0.4) and the shape operators of the immersion, that is, the operator $T_{p}$ defined in (2.2).

Theorem 2.3. Let $M^{n} \rightarrow \mathbf{R}^{d}$ be an isometric immersion with $M$ either closed or with a $p$-convex boundary. For all $p=1, \ldots, n-1$, one has

$$
\mu_{1, p}^{\prime \prime}(M)-\mu_{1, p}^{\prime}(M) \geq \frac{1}{p} \inf _{M}\left(W_{p}-T_{p}\right) .
$$

The notation on the right-hand side refers to the infimum over $x \in M$ of the lowest eigenvalue of $W_{p}-T_{p}$ acting on $p$-forms at $x$.

The inequality is sharp if $M=\mathbf{S}^{n}$, or $M$ is a hemisphere of $\mathbf{S}^{n}$, in which case $\mu_{1, p}^{\prime \prime}-\mu_{1, p}^{\prime}=n-2 p$.

The proof is given in Section 4.2 An immediate consequence of Theorem 2.3 is the following extrinsic condition for having no 1-gap. Recall that $\mu_{1,1}=\mu_{1,0}$ iff $\mu_{1,1}^{\prime \prime} \geq \mu_{1,1}^{\prime}$. In what follows, we assume that $M$ is either closed or has a convex boundary.

Corollary 2.4. (a) Let $M^{n} \rightarrow \mathbf{R}^{d}$ be an isometric immersion. If Ric $\geq T_{1}$ at all points of $M$, then $\mu_{1,1}(M)=\mu_{1,0}(M)$.

(b) Let $M^{n}$ be a convex hypersurface of $\mathbf{R}^{n+1}$, and assume that, at any point of $M$, any fixed principal curvature of $M$ is not greater than the sum of all the others. Then $\mu_{1,1}(M)=\mu_{1,0}(M)$.

In (b) we assume that the principal curvatures of $M$ are all nonnegative: this is possible by choosing appropriately the unit normal field $\nu$ on $M$. Part (b) then follows by expliciting the condition Ric $-S_{\nu}^{2} \geq 0$ in terms of the principal curvatures (by Gauss lemma, the condition is equivalent to $\operatorname{tr}\left(S_{\nu}\right) S_{\nu}-2 S_{\nu}^{2} \geq 0$ ).

Remark 2.5. For a compact Riemannian manifold the condition Ric $\geq 0$ alone does not guarantee that $\mu_{1,1}(M)=\mu_{1,0}(M)$.

In fact (see [2], Theorem 0.1 and Example 1.2) the Berger spheres (in the case of odd dimensions) or their products with $S^{1}$ (for even dimensions) provide a family of manifolds $\left\{M_{\epsilon}, \epsilon>0\right\}$ such that $\operatorname{Ric}_{M_{\epsilon}}>0$ for all $\epsilon, \mu_{1,0}\left(M_{\epsilon}\right)$ is bounded below by a positive number independent on $\epsilon$ and $\mu_{1,1}\left(M_{\epsilon}\right) \rightarrow 0$ as $\epsilon \rightarrow 0$.

Note that we do not assume any upper bound on the diameter of the hypersurfaces in (b). On the other hand, it is proved in 8 that for any convex $n$-dimensional hypersurface $M^{n}$ in $\mathbf{R}^{n+1}(n \geq 2)$ of diameter $d$, one has:

$$
\mu_{1, p}(M) \geq \frac{\max (1, p)}{2 e^{3}} \frac{1}{d^{2}} .
$$

This generalizes the inequality proved for functions by $\mathrm{Li}$ and Yau in [10].

We finally apply Theorem 2.3 to Euclidean and spherical domains. Recall that, for any Euclidean domain, one has the inequality $\mu_{1,0}(M)<\lambda_{1,0}(M)$. The examples of Section 1 show that there is no a priori order between $\mu_{1, p}(M)$ and $\mu_{1, p-1}(M)$. However, we have 
Theorem 2.6. (a) Assume that the Euclidean domain $M$ is p-convex for some $p=1, \ldots, n-1$. Then

$$
\mu_{1, p-1}(M) \leq \mu_{1, p}(M) \leq \cdots \leq \mu_{1, n}(M)=\lambda_{1,0}(M) .
$$

(b) If $M$ is actually convex, then the sequence $\left\{\mu_{1, p}\right\}$ is nondecreasing with respect to the degree $p$ :

$$
\mu_{1,0}(M)=\mu_{1,1}(M) \leq \mu_{1,2}(M) \leq \cdots \leq \mu_{1, n}(M)=\lambda_{1,0}(M),
$$

and, for all $p=0, \ldots,[n / 2]$, one has $\mu_{1, p}(M) \leq \lambda_{1, p}(M)$.

(c) If $M$ is strictly $p$-convex (that is, if $\sigma_{p}>0$ ), then $\mu_{1, q}(M)<\mu_{1, q+1}(M)$ for all $q \geq p$. Hence the inequalities in (a) are strict (starting from $\mu_{1, p}(M)$ ), and the inequalities in (b) are strict (starting from $\mu_{1,1}(M)$ ); in particular, for a strictly convex domain one has $\mu_{1, p}(M)<\lambda_{1, p}(M)$ for all $0 \leq p<n / 2$.

Proof. (a) Note that $\mu_{1, n} \geq \mu_{1, n-1}$ is always true by the Hodge decomposition (0.1) and (0.2). As a $p$-convex domain is $q$-convex for all $q \in[p, n-1]$, it is enough to show that $\mu_{1, p} \geq \mu_{1, p-1}$. Now, viewing $M$ as a totally geodesic hypersurface of $\mathbf{R}^{n+1}$ (so that $T_{p}=W_{p}=0$ ) we get, by Theorem 2.3, $\mu_{1, p}^{\prime \prime} \geq \mu_{1, p}^{\prime}$. Hence, by (0.2):

$$
\mu_{1, p}=\mu_{1, p}^{\prime}=\mu_{1, p-1}^{\prime \prime} \geq \mu_{1, p-1}
$$

and (a) follows. Part (b) is immediate from (a) and the Hodge duality. We prove (c). As $\sigma_{q} \geq \sigma_{p}$ for all $q \geq p$, it is enough to show that, if $p=1, \ldots, n-1$ and $\sigma_{p}>0$, then $\mu_{1, p+1}>\mu_{1, p}$.

Let $\omega$ be an eigenform associated to $\mu_{1, p}^{\prime \prime}$. We first show that

$$
\int_{\partial M}\|\omega\|^{2}>0
$$

that is, $\omega$ does not vanish identically on $\partial M$. Fix a coordinate system $\left(x_{1}, \ldots, x_{n}\right)$ on $\mathbf{R}^{n}$, and let $\phi=\omega\left(\frac{\partial}{\partial x_{i_{1}}}, \ldots, \frac{\partial}{\partial x_{i_{p}}}\right)$ be any component function of $\omega$ which does not vanish identically on $M$. Then $\phi$ integrates to zero on $M$ because $\omega$ is co-closed (see (4.1) in the proof of Lemma 4.7); if $\omega$ is identically zero on $\partial M$, then $\phi$ satisfies:

$$
\left\{\begin{array}{l}
\Delta \phi=\mu_{1, p}^{\prime \prime} \phi, \\
\phi_{\mid \partial M}=0,
\end{array}\right.
$$

and then $\mu_{1, p}^{\prime \prime} \geq \lambda_{1,0}$. However, as any first Dirichlet eigenfunction does not change sign, one must actually have $\mu_{1, p}^{\prime \prime} \geq \lambda_{2,0}>\lambda_{1,0}$, and this is impossible because otherwise:

$$
\mu_{1, p+1}=\mu_{1, p+1}^{\prime}=\mu_{1, p}^{\prime \prime}>\lambda_{1,0}
$$

contradicting part (a) of the theorem. Now, from the proof of Theorem 2.3:

$$
p\left(\mu_{1, p}^{\prime \prime}-\mu_{1, p}^{\prime}\right) \geq \sigma_{p} \int_{\partial M}\|\omega\|^{2}>0
$$

hence $\mu_{1, p}^{\prime \prime}>\mu_{1, p}^{\prime}$ and, in turn, $\mu_{1, p+1}>\mu_{1, p}$, as asserted.

Thus, for convex Euclidean domains, the first eigenvalue for $p$-forms, with either the absolute or relative conditions, belongs to the interval $\left[\mu_{1,0}, \lambda_{1,0}\right]$. Combining this fact with the Payne-Weinberger (13]) inequality on $\mu_{1,0}$, and using domain monotonicity for $\lambda_{1,0}$ one gets, for all $p=0, \ldots, n$ :

$$
\frac{\pi^{2}}{\operatorname{diam}(M)^{2}} \leq \mu_{1, p}(M) \leq \frac{j_{n}^{2}}{R^{2}},
$$


where $j_{n}^{2}$ is the first Dirichlet eigenvalue of the unit ball in $\mathbf{R}^{n}$ and $R$ is the inner radius of $M$. By Hodge duality, (2.4) holds also for $\lambda_{1, p}(M)$. Note that the following bound is obtained in [8], by different methods:

$$
\mu_{1, p}(M) \geq \frac{\max \{p(n-p), n-1\}}{n e^{3}} \frac{1}{\operatorname{diam}(M)^{2}} .
$$

Remark 2.7. Now let $M$ be any Euclidean domain, and $\bar{M}$ the ball with the same volume: the classical Weinberger isoperimetric inequality (see [18) states that $\mu_{1,0}(M) \leq \mu_{1,0}(\bar{M})$. As $\mu_{1,1}(M) \leq \mu_{1,0}(M)$, and $\mu_{1,0}(\bar{M})=\mu_{1,1}(\bar{M})$ (by Theorem 2.6 applied to balls), we can extend it to 1-forms:

$$
\mu_{1,1}(M) \leq \mu_{1,1}(\bar{M}) .
$$

For spherical domains, we have the following comparison result, proved in Section 4.2 .

Theorem 2.8. Let $M$ be a convex domain of $\mathbf{S}^{n}$, and $\mathbf{S}_{+}^{n}$ the hemisphere. Then

(a) For all $p=1, \ldots, n$ one has $\mu_{1, p}(M)-\mu_{1, p-1}(M) \geq \mu_{1, p}\left(\mathbf{S}_{+}^{n}\right)-\mu_{1, p-1}\left(\mathbf{S}_{+}^{n}\right)$.

(b) For all $p=0, \ldots,[n / 2]$ one has $\lambda_{1, p}(M) \geq \mu_{1, p}(M)$.

(c) The spectrum of the Laplacian on p-forms, for either the absolute or relative conditions, is bounded below by $\mu_{1,0}(M)$, that is, by the first Neumann eigenvalue of the Laplacian on functions. More precisely:

$$
\begin{aligned}
\mu_{1, p}(M)-\mu_{1,0}(M) \geq & \mu_{1, p}\left(\mathbf{S}_{+}^{n}\right)-\mu_{1,0}\left(\mathbf{S}_{+}^{n}\right) \\
& =\left\{\begin{array}{l}
0 \quad \text { if } p=1, n, \\
(p-1)(n-p) \text { if } 2 \leq p \leq[n / 2], \\
p(n-p-1) \text { if } 1+[n / 2] \leq p \leq n-1 .
\end{array}\right.
\end{aligned}
$$

Note that, unlike convex Euclidean domains, the sequence $\left\{\mu_{1, p}\right\}_{p=0, \ldots, n}$ may be decreasing at some $p$ : the $p$-gap of the hemisphere is nonnegative for $p \leq[n / 2]$, and nonpositive otherwise (see (4.13)). However, convex domains in $\mathbf{R}^{n}$ and $\mathbf{S}^{n}$ do share the following features: they have no 1-gap (i.e., $\mu_{1,1}=\mu_{1,0}$ ), their Hodge spectrum is bounded below by $\mu_{1,0}$, and, up to $p=[n / 2]$, their absolute first eigenvalues are not greater than the relative ones (Corollary 4.5 in Section 4.1 shows that this is not true in general).

Finally, we give a rough idea of the proof of the main inequality (Theorem 2.1). We consider, on the immersed manifold $M^{n}$, the family of all vector fields which are the orthogonal projection of unit parallel vector fields on the ambient Euclidean space $\mathbf{R}^{d}$. Note that this family is naturally parametrized by $\mathbf{S}^{d-1}$. Contracting the given $p$-form $\omega$ with any such field $V$ will reduce the degree by one and, with the given conditions on $\omega$, will produce a test form $i_{V} \omega$ for the eigenvalue $\mu_{1, p-1}^{\prime \prime}(M)=\mu_{1, p}^{\prime}(M)$. By the min-max principle, we obtain a family of inequalities indexed by $\mathbf{S}^{d-1}$; integrating these inequalities on $\mathbf{S}^{d-1}$ one gets Theorem 2.1 .

\section{A LOWER BOund For the Hodge Eigenvalues}

We give a lower bound of $\mu_{1, p}(M)$ when $M$ is a Riemannian manifold with $p$ convex (in particular, convex) boundary. We refer to [6] for estimates of the Hodge eigenvalues, valid also for manifolds with boundary.

We derive the lower bound by integrating the Bochner formula applied to an eigenform associated to $\mu_{1, p}(M)$ (which is a classical procedure). However, as 
$\partial M \neq \emptyset$, the term $\int_{M} \Delta\|\omega\|^{2}$ does not integrate to zero, and we wish to lower bound it by the principal curvatures, i.e., by $\sigma_{p}$ and $\int_{M}\|\omega\|^{2}$. Using Green's formula and the boundary conditions satisfied by the eigenform, one sees that the problem boils down to giving a lower bound of the ratio:

$$
\frac{\int_{\partial M}\|\omega\|^{2}}{\int_{M}\|\omega\|^{2}} \text {. }
$$

The main result of this section approaches this problem for any nonnegative function satisfying a suitable Laplacian condition. It is given in terms of a geometric constant $A$ (see Definition 4.11 in Section 4.3) which depends on a lower bound $(n-1) K$ of the Ricci curvature of $M$, a lower bound $H$ of the mean curvature of $\partial M$, and the inner radius $R$ of $M$. Under any of the following two conditions:

$$
K \geq 0, \text { or } K<0 \text { and } H \geq \sqrt{|K|}
$$

one can simply take $A=(n-1) H$. Then:

Theorem 3.1. Let $M$ be a Riemannian manifold with smooth boundary, and assume that the nonnegative function $\phi$ satisfies $\Delta \phi \leq \mu \phi$ on $M$, for some $\mu \in \mathbf{R}$. Fix any $t \in\left(0, \frac{\pi}{2 R}\right)$. Then, if $\mu \leq \frac{A^{2}}{4}+t^{2}$, one has:

$$
\int_{\partial M} \phi \geq\left[\frac{A}{2}+t \cot (R t)\right] \cdot \int_{M} \phi .
$$

The proof (given in Section 4.3) uses the distance function $\rho$ from the boundary of the manifold, and the so-called "mean-value lemma" (see [15]). In fact, any constant $A$ such that the inequality $\Delta \rho \geq A$ holds (in the sense of distributions on $M)$ will work.

The following corollary shows that Theorem 3.1 implies some well-known sharp lower bounds for the first Dirichlet eigenvalue $\lambda_{1,0}(M)$.

Corollary 3.2. Let $M$ be a Riemannian manifold with smooth boundary satisfying one of the conditions in (3.1). If $H \geq 0$, then:

$$
\lambda_{1,0}(M) \geq \frac{1}{4}(n-1)^{2} H^{2}+\frac{\pi^{2}}{4 R^{2}} .
$$

The corollary follows immediately by applying Theorem 3.1 to a first positive Dirichlet eigenfunction $\phi$, and by letting $t$ tend to $\pi /(2 R)$.

The bound is sharp in the following two cases. First, if $M=B_{R}^{n}$ is a geodesic ball in $\mathbf{H}^{n}$, then $H=\operatorname{coth} R>1=\sqrt{|K|}$ for all $R$; hence:

$$
\begin{aligned}
\lambda_{1,0}\left(B_{R}^{n}\right) & \geq \frac{1}{4}(n-1)^{2} \operatorname{coth}^{2} R+\frac{\pi^{2}}{4 R^{2}} \\
& >\frac{1}{4}(n-1)^{2},
\end{aligned}
$$

which is well known, and is sharp as $R \rightarrow \infty$ by a result of McKean 12. If $K=H=0$, the bound becomes

$$
\lambda_{1,0}(M) \geq \frac{\pi^{2}}{4 R^{2}},
$$

which is originally due to Li and Yau [10], and is sharp for flat cylinders, that is, for any manifold which is the Riemannian Cartesian product of a closed manifold and the interval $[0,2 R]$. 
Applying Theorem 3.1 to the square of the norm of an eigenform, one gets the desired lower bound for the Hodge eigenvalues. Recall the definition (0.5) of the constant $\sigma_{p}$ as a lower bound of the $p$-curvatures of $\partial M$ (note that $\sigma_{p} \geq p \sigma_{1}$ where $\sigma_{1}$ is a lower bound of the principal curvatures of $\left.\partial M\right)$.

Theorem 3.3. Let $M$ be a Riemannian manifold with smooth boundary, with curvature operator bounded below by $\gamma \in \mathbf{R}$, and $p$-curvatures of $\partial M$ bounded below by $\sigma_{p} \geq 0$.

(a) For all $t \in\left(0, \frac{\pi}{2 R}\right)$, and $p=1, \ldots, n-1$ :

$$
\mu_{1, p}(M) \geq \gamma p(n-p)+\min \left\{\frac{A^{2}}{8}+\frac{t^{2}}{2}, \sigma_{p}\left(\frac{A}{2}+t \cot (R t)\right)\right\},
$$

where the constant $A$ has been defined in (4.18).

(b) If $M$ satisfies one of the conditions in (3.1), then

$$
\mu_{1, p}(M)>\gamma p(n-p)+\frac{n-1}{2 p^{2}} \min \left\{\frac{n-1}{4}, p\right\} \cdot \sigma_{p}^{2} .
$$

In particular,

$$
\lambda_{1,1}(M)>(n-1) K+\frac{(n-1)^{2}}{8} H^{2}
$$

Proof. (a) Let $\omega$ be an eigenform associated to $\mu_{1, p}(M)$ having unit $L^{2}$-norm. We apply Bochner's formula (0.4) to $\omega$ : replacing the energy term by zero, and recalling that the curvature term $\left\langle W_{p}(\omega), \omega\right\rangle$ is bounded below by $\gamma p(n-p)\|\omega\|^{2}$, one gets:

$$
\Delta\left(\|\omega\|^{2}\right) \leq \mu\|\omega\|^{2}
$$

with $\mu=2 \mu_{1, p}(M)-2 \gamma p(n-p)$. We now apply Theorem 3.1 to $\phi=\|\omega\|^{2}$. Then, for any $t \in\left(0, \frac{\pi}{2 R}\right)$, either $\mu>\frac{A^{2}}{4}+t^{2}$, that is,

$$
\mu_{1, p}(M)>\gamma p(n-p)+\frac{A^{2}}{8}+\frac{t^{2}}{2}
$$

or else

$$
\int_{\partial M}\|\omega\|^{2} \geq \frac{A}{2}+t \cot (R t)
$$

If the second alternative holds, then integrating (3.2) over $M$ and using Lemma 4.10 in Section 4.2 one gets:

$$
\mu \geq \int_{M} \Delta\left(\|\omega\|^{2}\right) \geq 2 \sigma_{p} \int_{\partial M}\|\omega\|^{2} \geq 2 \sigma_{p}\left[\frac{A}{2}+t \cot (R t)\right],
$$

that is,

$$
\mu_{1, p}(M) \geq \gamma p(n-p)+\sigma_{p}\left(\frac{A}{2}+t \cot (R t)\right)
$$

which, together with (3.3), implies (a).

(b) We can take $A=(n-1) H$. We ignore the term in $t$ (depending on the inner radius) in the lower bound of part a) and observe that, by the definition of $\sigma_{p}$ as the sum of the lowest $p$ principal curvatures, one has $H \geq \sigma_{p} / p$. The second formula follows because, for $p=1, n-1$, the curvature term in Bochner's formula is just the Ricci curvature of $M$. 
Remark 3.4. For a p-convex Euclidean domain one has in particular:

$$
\mu_{1, p}(M)>\frac{1}{8} \sigma_{p}^{2}
$$

and, if the mean curvature of $\partial M$ is positive:

$$
\lambda_{1,1}(M) \geq \frac{(n-1)^{2}}{8} H^{2} .
$$

In some cases, (3.4) is better than the bounds (2.4) and (2.5), valid for convex domains and of type $\mu_{1, p}(M) \geq$ const/diam $(M)^{2}$. To see that consider, in $\mathbf{R}^{3}$, a thin cigar, that is, the domain $M_{\epsilon}$ bounded by a cylinder of unit length and small radius $\epsilon>0$, at the ends of which we glue two hemispheres of radius $\epsilon$. The boundary of $M_{\epsilon}$ can be smoothened so that, as $\epsilon$ tends to zero, the mean curvature, hence $\sigma_{2}\left(\partial M_{\epsilon}\right)$, tends to $\infty$; by (3.4), one then has

$$
\lim _{\epsilon \rightarrow 0} \mu_{1,2}\left(M_{\epsilon}\right)=\infty \text {. }
$$

On the other hand, the diameter of $M_{\epsilon}$ is always bounded below by 1 . Actually, taking the cylindrical part of length $1 / \epsilon$, one sees that (3.5) still holds and yet the diameter can be arbitrarily large.

We finally observe that in the case of negative curvature Theorem 3.3 sometimes gives a positive lower bound of $\lambda_{1, p}(M)$ which is independent of the inner radius (in particular, independent of the diameter). This is in contrast with the Euclidean space, where (2.4) shows that convex domains with large inner radius have small first Hodge eigenvalues.

It is well known that, for any domain $M$ in the hyperbolic space $\mathbf{H}^{n}$,

$$
\lambda_{1,0}(M)>\frac{(n-1)^{2}}{4},
$$

thus giving a lower bound independent of the inner radius of $M$ : (3.6) is true for geodesic balls (see Corollary 3.2 for another proof) and extends to any domain by the monotonicity of $\lambda_{1,0}$.

Such phenomenon cannot possibly hold for eigenvalues of $p$-forms $(p \geq 1)$ on arbitrary hyperbolic domains. In fact, the Euclidean domains $\Omega_{n-p, u}(p \geq 2)$ considered in Theorem 4.1 below, which are parametrized by $u \in\left(0, \frac{1}{10}\right)$, have diameter bounded above by 2 , and have their $\lambda_{1, p}$ tending to zero as $u \rightarrow 0$. Moreover, as $\lambda_{1,2}^{\prime \prime}\left(\Omega_{n-2, u}\right)$ is bounded below by a positive constant independent of $u$, $\lambda_{1,1}\left(\Omega_{n-2, u}\right)$ tends to zero with $u$. These domains are quasi-isometric to hyperbolic domains, say $\tilde{\Omega}_{n-p, u}, \tilde{\Omega}_{n-2, u}$ (with fixed constants because their diameters are bounded independently of $u$ ). By Proposition 3.3 of [3, the latter domains in $\mathbb{H}^{n}$ have the same small eigenvalues as the Euclidean ones.

Corollary 3.5 below does generalize (3.6) to $p$-forms, provided that $M$ is in the class of (convex) hyperbolic domains whose principal curvatures are bounded below by 1 (geodesic balls of arbitrary radius belong to that class) and that $p$ is not too far away from zero $(p \leq(n-2) / 8$ will be enough).

Corollary 3.5. Assume that the manifold $M^{n}$ has curvature operator bounded below by $\gamma<0$, and that $\sigma_{n-p} \geq(n-p) \sqrt{|\gamma|}$. If $n \geq n_{p}:=4 p+2+\sqrt{8 p^{2}+8 p}$, then:

$$
\lambda_{1, p}\left(M^{n}\right)>\left[\frac{1}{8}(n-1)^{2}-p(n-p)\right] \cdot|\gamma| \geq c_{p}(n-1)^{2} \cdot|\gamma|,
$$

where $c_{p}=\frac{1}{8}-p \frac{n_{p}-p}{\left(n_{p}-1\right)^{2}}$ is positive and depends only on $p$. 
In particular, if the Ricci curvature of $M$ is bounded below by $(n-1) K$, with $K<0$, and the mean curvature of its boundary is bounded below by $\sqrt{|K|}$, then, for all dimensions $n \geq 10$, one has

$$
\lambda_{1,1}(M)>\frac{1}{72}(n-1)^{2} \cdot|K| .
$$

Remark 3.6. The boundary curvature condition is certainly satisfied if $\partial M$ is convex, with principal curvatures bounded below by $\sqrt{|\gamma|}$.

Note also the asymptotic lower bound $\lambda_{1, p} \geq \frac{1}{8} n^{2}|\gamma|$ as $n$ tends to $\infty$; that is, given $\epsilon>0$ and a positive integer $p$ there exists $n(\epsilon, p)$ such that

$$
\frac{\lambda_{1, p}\left(M^{n}\right)}{n^{2}}>\left(\frac{1}{8}-\epsilon\right)|\gamma|
$$

holds for all manifolds satisfying the assumptions of the corollary and having dimension $n \geq n(\epsilon, p)$.

Remark 3.7. The dimensional restriction $n \geq n_{p}$ is probably not sharp; however, it is somewhat justified by the fact that, for all $n$ (see [1], Theorem II.5, p. 46),

$$
\lim _{R \rightarrow \infty} \lambda_{1, n}\left(B_{R}^{n}\right)=\lim _{R \rightarrow \infty} \mu_{1,0}\left(B_{R}^{n}\right)=0,
$$

where $B_{R}^{n}$ denotes the geodesic ball of radius $R$ in $\mathbf{H}^{n}$.

This fact shows that Corollary 3.5 cannot hold for all $n$ and $p$.

Proof of the corollary. First, let us observe that $M$ satisfies the conditions in (3.1). In fact, if $K<0$, one has $|\gamma| \geq|K|$ because $\gamma \leq K$; hence

$$
H \geq \sigma_{n-p} /(n-p) \geq|\gamma| \geq|K| .
$$

We now apply Theorem 3.3 (b); if $n \geq n_{p}$ then in particular $(n-1) / 4 \leq n-p$ and

$$
\begin{aligned}
\lambda_{1, p}(M) & =\mu_{1, n-p}(M) \\
& >\left[-p(n-p)+\frac{n-1}{2} \cdot \min \left\{\frac{n-1}{4}, n-p\right\}\right] \cdot|\gamma| \\
& =\left[-p(n-p)+\frac{1}{8}(n-1)^{2}\right] \cdot|\gamma| .
\end{aligned}
$$

One easily checks that the right-hand side is positive for all $n \geq n_{p}$. Then

$$
\begin{aligned}
\lambda_{1, p}(M) & >(n-1)^{2}\left[\frac{1}{8}-p \frac{n-p}{(n-1)^{2}}\right]|\gamma| \\
& \geq(n-1)^{2}\left[\frac{1}{8}-p \frac{n_{p}-p}{\left(n_{p}-1\right)^{2}}\right]|\gamma| \\
& =c_{p}(n-1)^{2}|\gamma| .
\end{aligned}
$$

\section{Proofs of the main theorems}

4.1. Proof of Theorem 1.1. We start by restating Theorem 2.1 of 9 . In what follows, $u$ is a real parameter in $\left(0, \frac{1}{10}\right)$ and $c_{1}, c_{2}, \ldots$ are positive constants not depending on $u$. 


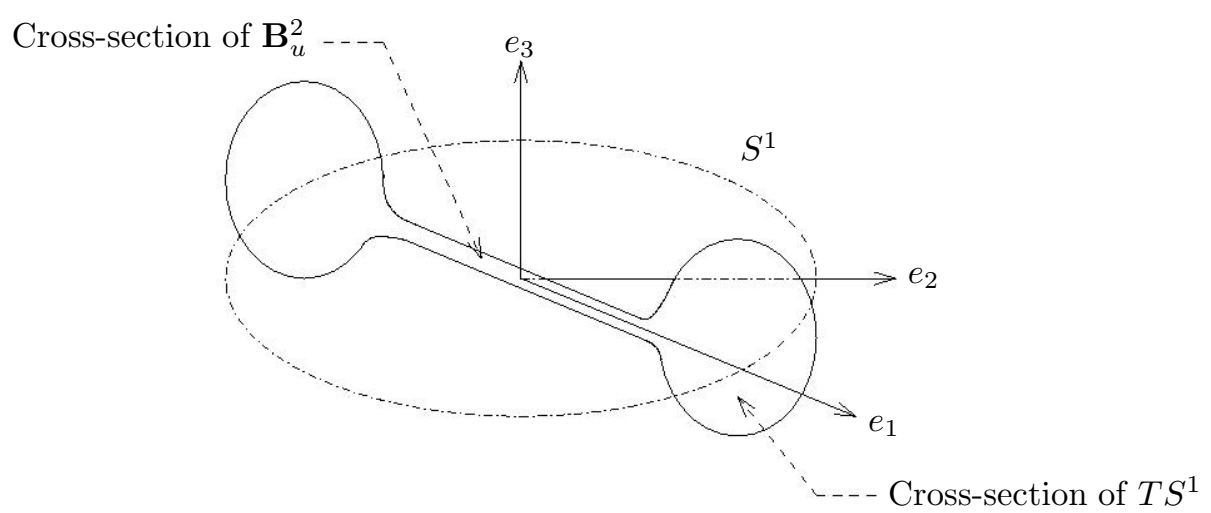

Figure 1. Cross-section of $\Omega_{1, u} \subset \mathbb{R}^{3}$ with a plane containing $e_{3}$.

Theorem 4.1. Let $n \geq 2$ and $p=0, \ldots, n-2$. There exists a family $\Omega_{p, u}$ of domains of $\mathbf{R}^{n}$, diffeomorphic to balls, of diameter bounded above by 2 , such that

$$
\lim _{u \rightarrow 0} \mu_{1, p}^{\prime \prime}\left(\Omega_{p, u}\right)=0 \quad \text { and } \quad \mu_{1, q}^{\prime \prime}\left(\Omega_{p, u}\right) \geq c_{1} \quad \text { for all } u \in\left(0, \frac{1}{10}\right) \text { and } p \neq q .
$$

The domains $\Omega_{p, u}$ generalize the classical Cheeger's dumbbell balls (made of two Euclidean balls linked together by a cylinder of small radius). They consist of a "thick" part, independent of $u$, and a "thin" part which will collapse to a $(p+1)$-dimensional ball as $u \rightarrow 0$. Let us see their construction in detail.

Fix $u \in\left(0, \frac{1}{10}\right)$, and split $\mathbf{R}^{n}=\mathbf{R}^{p+1} \times \mathbf{R}^{n-p-1}$. Let $\mathbf{S}^{p}$ the unit sphere in $\mathbf{R}^{p+1}$ (centered at the origin). Define:

$$
\begin{aligned}
T \mathbf{S}^{p} & :=\text { thick part of } \Omega_{p, u} \\
& =1 / 2 \text {-tubular neighborhood of } \mathbf{S}^{p} \text { in } \mathbf{R}^{n}
\end{aligned}
$$

and

$$
\begin{aligned}
\mathbf{B}_{u}^{p+1} & :=\text { thin part of } \Omega_{p, u} \\
& =u \text {-tubular neighborhood of } \mathbf{B}^{p+1}\left(\frac{1}{2}\right) \subseteq \mathbf{R}^{p+1} \text { in } \mathbf{R}^{n} .
\end{aligned}
$$

Note that $\mathbf{B}^{p+1}\left(\frac{1}{2}\right)$ is the "central hole" of $T \mathbf{S}^{p}$.

To get $\Omega_{p, u}$, we now smoothly glue the thick part and the thin part together, along a $2 u$-tubular neighborhood of $\mathbf{S}^{p}\left(\frac{1}{2}\right)=\partial \mathbf{B}^{p+1}\left(\frac{1}{2}\right) \subseteq \partial T \mathbf{S}^{p}$.

Clearly, the resulting domain is diffeomorphic to a ball for all $u$; as $u \rightarrow 0$, it collapses to the singular manifold $T \mathbf{S}^{p} \# \mathbf{B}^{p+1}\left(\frac{1}{2}\right)$ (glued along $\mathbf{S}^{p}\left(\frac{1}{2}\right)$ ).

When $p=1$ and $n=3$, the thick part is a full immersed torus in $\mathbf{R}^{3}$ of radii $1,1 / 2$, and the thin part is a $u$-tubular neighborhood of the 2 -disk of radius $1 / 2$. Here is a cross-section of $\Omega_{1, u}$ (Figure 1); the full domain $\Omega_{1, u}$ is obtained by rotation of it around the axis $e_{3}$.

The small eigenvalue of Theorem 4.1 comes from a harmonic $p$-form on $\mathbf{S}^{p}$.

Now let $M$ be a manifold with boundary. For $p=0, \ldots, n-2$ and $u \in\left(0, \frac{1}{10}\right)$, we construct the following family of Riemannian manifolds $M \# \Omega_{p, u}^{\prime}$, diffeomorphic to $M$, which will be used in the proof of the theorem: the metrics there will be those of $M \# \Omega_{p, u}^{\prime}$ for a suitable $p$ and for $u$ sufficiently small. 


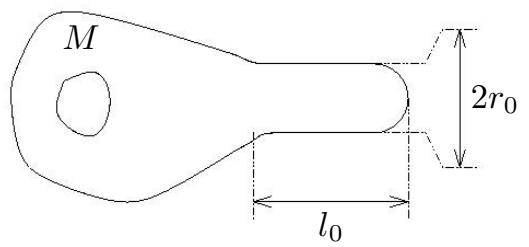

Figure 2. Metric with a "bump" on $M$.

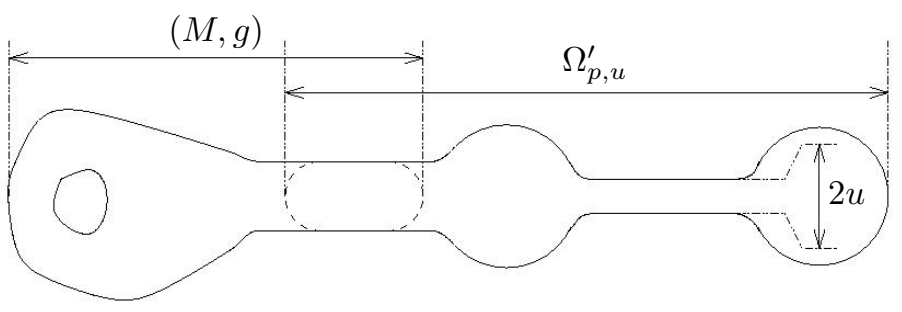

Figure 3. Covering of $M \# \Omega_{p, u}^{\prime}$.

Let $r_{0}, l_{0}$ be two fixed, positive real numbers $\left(r_{0}<l_{0} / 10\right)$, small enough to guarantee that the constructions below make sense. First, we smoothly glue, in a neighborhood of a point $m \in \partial M$, the manifold $M$ with the Euclidean cylinder $C\left(r_{0}, l_{0}\right):=B^{n-1}\left(r_{0}\right) \times\left[0, l_{0}\right]$ of radius $r_{0}$ and length $r_{0}$, suitably smoothened so that we obtain a smooth bump near $m$; see Figure 2 ,

As the resulting manifold is diffeomorphic to the original one, we can assume from the start that the metric $g$ on $M$ has the desired bump.

Next, we glue another copy of the cylinder $C\left(r_{0}, l_{0}\right)$ with $\Omega_{p, u}$ in a neighborhood of its thick part, and obtain the Euclidean domain $\Omega_{p, u}^{\prime}$.

Remark 4.2. As $\Omega_{p, u}^{\prime}$ is quasi-isometric to $\Omega_{p, u}$ with quasi-isometry constants independent of $u$, the spectral properties of Theorem 4.1 valid for $\Omega_{p, u}$, continue to hold for $\Omega_{p, u}^{\prime}$ thanks to [3], Prop. 3.3.

Finally, we glue $(M, g)$ with $\Omega_{p, u}^{\prime}$ by identifying the final halves $B^{n-1}\left(r_{0}\right) \times$ $\left[l_{0} / 2, l_{0}\right]$ of the respective cylindrical bumps (note that the metrics are the same on the identified parts).

We denote the resulting Riemannian manifold with boundary by $M \# \Omega_{p, u}^{\prime}$ : it has a Euclidean cylindrical handle of length $3 l_{0} / 2$ and radius $r_{0}$. Moreover, it comes with a natural covering $\left\{U_{u}^{1}, U^{2}\right\}$, where $U_{u}^{1}=\Omega_{p, u}^{\prime}$ and $U^{2}=(M, g)$. Note that $U_{u}^{1} \cap U^{2}$ is basically a Euclidean cylinder of radius $r_{0}$ and length $l_{0} / 2$; as such, it is diffeomorphic to a ball in $\mathbf{R}^{n}$, and has volume bounded below independently of $u$. See Figure 3 Clearly $M \# \Omega_{p, u}^{\prime}$ is diffeomorphic to $M$ for all $u \in\left(0, \frac{1}{10}\right)$.

The following lemma shows that the manifolds $M \# \Omega_{p, u}^{\prime}$ and $\Omega_{p, u}$ have similar spectral properties.

Lemma 4.3. Let $p, q \in\{0, \ldots, n-2\}$. The manifolds $M \# \Omega_{p, u}^{\prime}$ satisfy:

a) $\lim _{u \rightarrow 0} \mu_{1, p}\left(M \# \Omega_{p, u}^{\prime}\right)=0$.

b) If $q \geq 1$ and $q \neq p$, then $\mu_{1, q}^{\prime \prime}\left(M \# \Omega_{p, u}^{\prime}\right) \geq c_{2}$ for all $u$.

c) If $p \neq 0$, then $\mu_{1,0}\left(M \# \Omega_{p, u}^{\prime}\right) \geq c_{3}$ for all $u$. 
Proof. a) Let $b_{p}(M)$ be the $p$ th Betti number of the absolute cohomology of $M$ (hence of $M \# \Omega_{p, u}^{\prime}$ ). For $u$ small, we construct an orthonormal family of $b_{p}(M)+1$ test $p$-forms on $M \# \Omega_{p, u}^{\prime}$ whose Rayleigh quotient tends to zero with $u$. The assertion will then follow from the min-max principle.

We consider the Riemannian manifold $M \# T^{\prime} \mathbf{S}^{p}$, glued as before from $(M, g)$ and the thick part $T \mathbf{S}^{p}$ of $\Omega_{p, u}$ along a common cylinder $C\left(r_{0}, l_{0}\right)$. Note that $M \# \Omega_{p, u}^{\prime}$ is obtained by gluing $M \# T^{\prime} \mathbf{S}^{p}$ with the thin part $\mathbf{B}_{u}^{p+1}$ near the $p$-sphere $\partial \mathbf{B}^{p+1}\left(\frac{1}{2}\right)$. An application of the Mayer-Vietoris sequence shows that $M \# T^{\prime} \mathbf{S}^{p}$ has $b_{p}(M)+1$ harmonic $p$-forms for the absolute boundary condition. Fix $u>0$ and small. We extend these harmonic forms by zero to the thin part $\mathbf{B}_{u}^{p+1}$, by using smooth cut-off functions which are constant outside a $2 u$-tubular neighborhood $W_{u}$ of $\partial \mathbf{B}^{p+1}\left(\frac{1}{2}\right)$. By a Gram-Schmidt procedure, we thus obtain an orthonormal family of $b_{p}(M)+1$ test $p$-forms on $M \# \Omega_{p, u}^{\prime}$, which are harmonic outside $W_{u}$. Let $u \rightarrow 0$; as $W_{u}$ retracts to the $p$-sphere $\partial \mathbf{B}^{p+1}\left(\frac{1}{2}\right)$, which is of zero capacity because it has codimension at least 2 in the manifold (recall that $p \leq n-2$ by assumption), the Rayleigh quotient of any of these forms tends to zero with $u$, as asserted.

b) We cover the manifold $M \# \Omega_{p, u}^{\prime}$ by $\left\{U_{u}^{1}, U^{2}\right\}$, where $U_{u}^{1}=\Omega_{p, u}^{\prime}, U^{2}=(M, g)$. By the McGowan lemma (11], Lemma 2.3, and [7, Lemma 1), as $U_{u}^{1} \cap U^{2}$ does not depend on $u$ and is diffeomorphic to a ball in $\mathbf{R}^{n}$, one has:

$$
\mu_{1, q}^{\prime \prime}\left(M \# \Omega_{p, u}^{\prime}\right) \geq c_{4} \cdot\left[\frac{1}{\mu_{1, q}^{\prime \prime}\left(\Omega_{p, u}^{\prime}\right)}+\frac{1}{\mu_{1, q}^{\prime \prime}(M, g)}\right]^{-1} \cdot\left[c_{5}+\frac{c_{6}}{\mu_{1, q}^{\prime \prime}\left(U_{u}^{1} \cap U^{2}\right)}\right]^{-1}
$$

for positive constants $c_{i}$ independent of $u$. By Remark 4.2 and Theorem 4.1 $\mu_{1, q}^{\prime \prime}\left(\Omega_{p, u}^{\prime}\right)$ is bounded below by a constant independent of $u$, and the assertion follows.

c) We will assume that there exists a sequence $u_{m} \rightarrow 0$ such that

$$
\lim _{m \rightarrow \infty} \mu_{1,0}\left(M \# \Omega_{p, u_{m}}^{\prime}\right)=0
$$

and get a contradiction.

We cover the manifold $M \# \Omega_{p, u}^{\prime}$ by $\left\{U_{u}^{1}, U^{2}\right\}$, where $U_{u}^{1}=\Omega_{p, u}^{\prime}, U^{2}=(M, g)$ as before. For all $m \in \mathbf{N}$ we choose an eigenfunction $f_{m}$ for the eigenvalue $\mu_{1,0}$ of unit $L^{2}$-norm, and let $f_{1, m}$ (resp. $\left.f_{2, m}\right)$ be the restriction of $f_{m}$ to $\Omega_{p, u_{m}}^{\prime}(\operatorname{resp} .(M, g))$. If $a_{1, m}$ is the mean value of $f_{1, m}$ on $\Omega_{p, u_{m}}^{\prime}$, one has, by the min-max principle:

$$
\begin{aligned}
\mu_{1,0}\left(\Omega_{p, u_{m}}^{\prime}\right) \int_{\Omega_{p, u_{m}}^{\prime}}\left|f_{1, m}-a_{1, m}\right|^{2} & \leq \int_{\Omega_{p, u_{m}}^{\prime}}\left\|d f_{1, m}\right\|^{2} \\
& \leq \int_{M \# \Omega_{p, u_{m}}^{\prime}}\left\|d f_{m}\right\|^{2} .
\end{aligned}
$$

But $\mu_{1,0}\left(\Omega_{p, u_{m}}^{\prime}\right) \geq c_{7}$ for all $m$ by Remark 4.2 and Theorem 4.1, while the last integral tends to zero as $m \rightarrow \infty$ by assumption. Therefore,

$$
\lim _{m \rightarrow \infty} \int_{\Omega_{p, u_{m}}^{\prime}}\left|f_{1, m}-a_{1, m}\right|^{2}=0 .
$$


Similarly, if $a_{2, m}$ is the mean value of $f_{2, m}$ on $U^{2}=(M, g)$, one has

$$
\lim _{m \rightarrow \infty} \int_{(M, g)}\left|f_{2, m}-a_{1, m}\right|^{2}=0 .
$$

By the triangle inequality

$$
\begin{aligned}
\left|a_{1, m}-a_{2, m}\right| & \cdot \operatorname{vol}\left(U_{u_{m}}^{1} \cap U^{2}\right)^{1 / 2} \\
& \leq\left\{\int_{U_{u_{m}}^{1} \cap U^{2}}\left|f_{1, m}-a_{1, m}\right|^{2}\right\}^{1 / 2}+\left\{\int_{U_{u_{m}}^{1} \cap U^{2}}\left|f_{2, m}-a_{2, m}\right|^{2}\right\}^{1 / 2} \\
& \leq\left\{\int_{\Omega_{p, u_{m}}^{\prime}}\left|f_{1, m}-a_{1, m}\right|^{2}\right\}^{1 / 2}+\left\{\int_{(M, g)}\left|f_{2, m}-a_{2, m}\right|^{2}\right\}^{1 / 2} .
\end{aligned}
$$

As $m \rightarrow \infty$, the right-hand side tends to zero; on the other hand, $\operatorname{vol}\left(U_{u_{m}}^{1} \cap U^{2}\right)$ does not depend on $m$. Hence $\left|a_{1, m}-a_{2, m}\right|$ tends to zero, which implies that also $\left\|f_{m}-a_{1, m}\right\|_{L^{2}}$ tends to zero. As all functions $f_{m}$ have unit $L^{2}$-norm, this contradicts their orthogonality to constant functions. The proof is complete.

We can now give the proof of Theorem 1.1.

Proof of Theorem 1.1. (1) Fix $p=0, \ldots, n-3$ and consider the manifold $M \# \Omega_{p+1, u}^{\prime}$. By Lemma $4.3 \mathrm{a}$ ), $\mu_{1, p+1}$ tends to zero with $u$. We prove that $\mu_{1, p} \geq c_{8}$ for all $u$. This is immediately true if $p=0$ by Lemma $4.3 \mathrm{c}$ ); if $p \geq 1$, by Lemma $4.3 \mathrm{~b}$ ), both $\mu_{1, p}^{\prime}=\mu_{1, p-1}^{\prime \prime}$ and $\mu_{1, p}^{\prime \prime}$ are bounded below by a constant for all $u$, hence so is $\mu_{1, p}$. Hence the desired metric $g_{p}$ is that of $M \# \Omega_{p+1, u}^{\prime}$ for $u$ sufficiently small.

(2) Fix $p=1, \ldots, n-1$ and consider the manifold $M \# \Omega_{p-1, u}^{\prime}$. For all $u$ both $\mu_{1, p+1}^{\prime}=\mu_{1, p}^{\prime \prime}$ and $\mu_{1, p+1}^{\prime \prime}$ are bounded below by a constant; then so is $\mu_{1, p+1}$. We show that

$$
\lim _{u \rightarrow 0} \mu_{1, p}=0
$$

so that the metric $g_{p}^{\prime}$ is that of $M \# \Omega_{p-1, u}^{\prime}$ for $u$ sufficiently small. As $\mu_{1,1} \leq \mu_{1,0}$, which tends to zero by Lemma $4.3 \mathrm{a}$ ), the above is true for $p=1$. Let $p=$ $2, \ldots, n-1$. Note that $\mu_{1, p-1}^{\prime}=\mu_{1, p}^{\prime \prime}$ is bounded below by a constant by Lemma $4.3 \mathrm{~b}$ ), and $\mu_{1, p-1}$ tends to zero. Hence $\mu_{1, p-1}^{\prime \prime}=\mu_{1, p-1}$ tends to zero, and so does $\mu_{1, p}^{\prime}$ and, a fortiori, $\mu_{1, p}$.

(3) Fix $p=0, \ldots, n-2$ and consider $M \# \Omega_{p, u}^{\prime}$. We show that, for small $u$ :

$$
\mu_{1, p}\left(M \# \Omega_{p, u}^{\prime}\right)=\mu_{1, p+1}\left(M \# \Omega_{p, u}^{\prime}\right) .
$$

First, observe that $\mu_{1, p}$ tends to zero. Let $u$ be small. If $p=0$, one has $\mu_{1,1}=\mu_{1,0}$ because $\mu_{1,1}^{\prime \prime}$ is bounded below by a constant. If $p=1, \ldots, n-2$, then by Lemma 4.3 b) both $\mu_{1, p}^{\prime}=\mu_{1, p-1}^{\prime \prime}$ and $\mu_{1, p+1}^{\prime \prime}$ are bounded below by a constant. Hence $\mu_{1, p}=\mu_{1, p}^{\prime \prime}=\mu_{1, p+1}^{\prime}=\mu_{1, p+1}$ and the proof is complete.

The proof for Euclidean and spherical metrics. Assume that we start from a Euclidean domain $M$; then it is clear that the metric of $M \# \Omega_{p, u}^{\prime}$ can be made Euclidean. If $M$ is a domain in $\mathbf{S}^{n}$, let $s$ be the stereographic projection from a point $x_{0}$ outside $M$. As the diameter of $\Omega_{p, u}$ is always bounded above by 2 (hence, using homotheties, can be assumed as small as necessary), we can find $x_{0}$ and $\epsilon>0$ so that none of the spherical domains $s^{-1}\left(s(M) \# \Omega_{p, u}^{\prime}\right)$ will intersect the ball of radius $\epsilon$ around $x_{0}$. As the projection $s$, restricted to the complement of that ball, is a 
quasi-isometry with fixed constants, Lemma 4.3 continues to hold for the Riemannian manifolds $s^{-1}\left(s(M) \# \Omega_{p, u}^{\prime}\right)$ (all diffeomorphic to $M$ and with spherical metric), and we carry on the proof of Theorem 1.1 as before.

We give two more consequences of Theorem 4.1 and Lemma 4.3.

Corollary 4.4. Let $M$ be a compact manifold with smooth boundary, and $p=$ $1, \ldots, n-2$. Then there exist metrics $h_{p}, h^{\prime}$ on $M$ such that:

a) $\mu_{1,0}\left(M, h_{p}\right)>\mu_{1, p}\left(M, h_{p}\right)$.

b) $\mu_{1,0}\left(M, h^{\prime}\right)<\mu_{1, p}\left(M, h^{\prime}\right)$ for all $p \neq 1$.

Proof. Just take, for $u$ sufficiently small, $\left(M, h_{p}\right)=M \# \Omega_{p, u}^{\prime}$ and $\left(M, h^{\prime}\right)=$ $M \# \Omega_{0, u}^{\prime}$ and proceed as before.

Corollary 4.5. Let $n \geq 4$ and $0<p<n / 2$. Then there exist domains in $\mathbf{R}^{n}$ (and also in $\mathbf{S}^{n}$ ) such that $\mu_{1, p}>\lambda_{1, p}$.

Remark 4.6. The assertion cannot be true for $p=0, n / 2$. In fact, it is well known that, for all Euclidean domains, $\mu_{1,0}<\lambda_{1,0}$, and for all manifolds, $\mu_{1, n / 2}=\lambda_{1, n / 2}$ when $n$ is even, by Hodge duality. The case $n=3, p=1$ is open.

In the next section we prove that, for convex Euclidean or spherical domains, $\mu_{1, p} \leq \lambda_{1, p}$ for $0 \leq p \leq n / 2$ and $n \geq 3$.

Proof of Corollary 4.5. Euclidean domains. First case: $0<p<\frac{n-1}{2}$. As $n-p-1 \leq$ $n-2$, we can take $\Omega=\Omega_{n-p-1, u}$ for $u$ small. In fact, $\mu_{1, n-p-1}^{\prime \prime} \rightarrow 0$, which implies $\lambda_{1, p}^{\prime \prime} \rightarrow 0$ hence $\lambda_{1, p} \rightarrow 0$. As $p<\frac{n-1}{2}$, both $\mu_{1, p}^{\prime}=\mu_{1, p-1}^{\prime \prime}$ and $\mu_{1, p}^{\prime \prime}$ are bounded below by a constant independent of $u$.

Second case: $n \geq 5$ odd, $p=\frac{n-1}{2}$. We can take $\Omega=\Omega_{\frac{n+1}{2}, u}$ for $u$ small $\left(\frac{n+1}{2} \leq n-2\right.$ because $\left.n \geq 5\right)$. In fact, $\mu_{1, \frac{n+1}{2}}^{\prime \prime} \rightarrow 0$ implies $\lambda_{1, \frac{n-1}{2}} \rightarrow 0$. One verifies immediately that both $\mu_{1, \frac{n-1}{2}}^{\prime}=\mu_{1, \frac{n-3}{2}}^{\prime \prime}$ and $\mu_{1, \frac{n-1}{2}}^{\prime \prime}$ are bounded below by a constant.

Spherical domains: apply the stereographic projection to Theorem 4.1 and proceed as in the Euclidean case.

\subsection{Proofs of Theorems 2.1, 2.3 and 2.8.}

Proof of Theorem 2.1. Let $M^{n} \rightarrow \mathbf{R}^{d}$ be an isometric immersion and $\omega$ a co-closed $p$-form on $M$, with $p=1, \ldots, n$. If $\partial M \neq \emptyset$, we assume in addition that $\omega$ is tangential on $\partial M$, that is, it vanishes whenever one of its arguments is a vector normal to the boundary. We have to prove that

$$
\int_{M}\left\{\|\nabla \omega\|^{2}+\left\langle T_{p}(\omega), \omega\right\rangle+(p-1)\|d \omega\|^{2}\right\} \geq p \mu_{1, p}^{\prime}(M) \int_{M}\|\omega\|^{2} .
$$

We consider the family of all vector fields on $\mathbf{R}^{d}$ which are parallel and have (constant) unit norm; this family is naturally identified with $\mathbf{S}^{d-1}$, and its typical element will be denoted by $\bar{V}$. At any point of $M$, we can split:

$$
\bar{V}=V+V^{\perp},
$$

where $V \in T M$ is the orthogonal projection of $\bar{V}$ onto $T M$ and $V^{\perp} \in T M^{\perp}$. Hence any element $\bar{V} \in \mathbf{S}^{d-1}$ will give rise to a vector field $V$ on $M$. We contract a given $p$-form $\omega$ on $M$ with such vector fields and obtain the following lemma. 
Lemma 4.7. Let $\omega \in \Lambda^{p}(M), p=1, \ldots, n$, be a co-closed form such that $i_{\tilde{\nu}} \omega=0$ on $\partial M$, where $\tilde{\nu}$ is the inner unit normal of $\partial M$. Then, for all $\bar{V} \in \mathbf{S}^{d-1}$,

$$
\mu_{1, p}^{\prime}(M) \int_{M}\left\|i_{V} \omega\right\|^{2} \leq \int_{M}\left\|d i_{V} \omega\right\|^{2} .
$$

We wish to integrate the inequalities of Lemma 4.7 with respect to $\bar{V} \in \mathbf{S}^{d-1}$ and the canonical measure of $\mathbf{S}^{d-1}$, which we denote by $d \bar{V}$. To that purpose, the following calculation holds.

Lemma 4.8. Let $\omega \in \Lambda^{p}(M)$ with $p=1, \ldots, n$. Then, at all points of $M$,

$$
\begin{aligned}
\int_{S^{d-1}}\left\|i_{V} \omega\right\|^{2} d \bar{V} & =c_{d} p\|\omega\|^{2}, \\
\int_{S^{d-1}}\left\|d i_{V} \omega\right\|^{2} d \bar{V} & =c_{d}\left\{\|\nabla \omega\|^{2}+\left\langle T_{p}(\omega), \omega\right\rangle+(p-1)\|d \omega\|^{2}\right\},
\end{aligned}
$$

where $c_{d}=\frac{\left|\mathbf{S}^{d-1}\right|}{d}$.

The theorem will follow by integrating the inequalities of Lemma 4.7 with respect to $\bar{V} \in \mathbf{S}^{d-1}$ and by using Lemma 4.8. It then remains to prove the two lemmas.

Proof of Lemma 4.7. We use the min-max principle. First observe that, for any $\bar{V} \in \mathbf{S}^{d-1}$, the vector field $V$ on $M$ is a gradient; precisely: $V=\nabla f_{V}$ where $f_{V}$ is the restriction to $M$ of the linear function on $\mathbf{R}^{d}$ given by the distance from any hyperplane orthogonal to $\bar{V}$. If $\omega \in \Lambda^{p}(M)$ and $f \in C^{\infty}(M)$, one has $\delta(f \omega)=-i_{\nabla f} \omega+f \delta \omega$, which shows that, if $\omega$ is co-closed, then $i_{V} \omega=-\delta\left(f_{V} \omega\right)$ is co-exact. Now assume that $M$ has nonempty boundary. If $p \geq 2$ and $\omega$ is tangential, then so is $i_{V} \omega$. Hence, for all $\bar{V} \in \mathbf{S}^{n-1}, i_{V} \omega$ is a tangential, co-exact ( $\left.p-1\right)$-form. As the test forms for the absolute eigenvalue problem need only satisfy $i_{\tilde{\nu}} \omega=0$ (which is our case), we can apply the min-max principle to $i_{V} \omega$. Then:

$$
\mu_{1, p-1}^{\prime \prime}(M) \int_{M}\left\|i_{V} \omega\right\|^{2} \leq \int_{M}\left\|d i_{V} \omega\right\|^{2},
$$

and the lemma follows because, by the Hodge decomposition, $\mu_{1, p-1}^{\prime \prime}(M)=\mu_{1, p}^{\prime}(M)$. If $p=1$ then $i_{V} \omega$ is a function; since

$$
\int_{M} i_{V} \omega=-\int_{M} \delta\left(f_{V} \omega\right)=-\int_{\partial M} f_{V} \omega(\tilde{\nu})=0
$$

$i_{V} \omega$ will be a test function for the Neumann problem. As $\mu_{1,0}(M)=\mu_{1,1}^{\prime}(M)$, the assertion is true also for $p=1$.

In what follows, all calculations hold at an arbitrary fixed point $x \in M$. We fix, around $x$, a local orthonormal frame $\left(e_{1}, \ldots, e_{n}\right)$ of $T M$ which is geodesic at $x$ : $\nabla_{e_{i}} e_{j}(x)=0$ for all $i, j=1, \ldots, n$, and a local orthonormal frame $\left(\nu_{1}, \ldots, \nu_{m}\right)$ of $T M^{\perp}$. Then we can write $V=\sum_{i=1}^{n}\left\langle\bar{V}, e_{i}\right\rangle e_{i}$ and $V^{\perp}=\sum_{\alpha=1}^{m}\left\langle\bar{V}, \nu_{\alpha}\right\rangle \nu_{\alpha}$. Before proving Lemma 4.8, we observe the following well-known fact. In what follows, $c_{d}=\frac{\left|\mathbf{S}^{d-1}\right|}{d}$.

Lemma 4.9. (a) If $\bar{X}, \bar{Y} \in T_{x} \mathbf{R}^{d}$, then $\int_{\mathbf{S}^{d-1}}\langle\bar{V}, \bar{X}\rangle\langle\bar{V}, \bar{Y}\rangle d \bar{V}=c_{d}\langle\bar{X}, \bar{Y}\rangle$.

(b) If $\omega, \phi \in \Lambda^{p}(M)$, then, at any point of $M$ one has $\int_{S^{d-1}}\left\langle i_{V} \omega, i_{V} \phi\right\rangle d \bar{V}=$ $c_{d} p\langle\omega, \phi\rangle$. 
Proof of Lemma 4.9. To prove (a) just fix a coordinate system and compute. (b) follows from (a) and the definition of inner product of forms.

Proof of Lemma 4.8 . The first equality is an immediate consequence of Lemma4.9 To prove the second, we consider the Lie derivative $L_{V} \omega$ of the $p$-form $\omega$ along the vector field $V$, and recall that $L_{V} \omega=d i_{V} \omega+i_{V} d \omega$. Hence:

$$
\left\|d i_{V} \omega\right\|^{2}=\left\|L_{V} \omega\right\|^{2}+\left\|i_{V} d \omega\right\|^{2}-2\left\langle i_{V} d \omega, L_{V} \omega\right\rangle .
$$

Lemma 4.8 will follow from (4.2) and the following identities, which are valid pointwise on $M$ :

Fact 1. $\int_{\mathbf{S}^{d-1}}\left\|L_{V} \omega\right\|^{2} d \bar{V}=c_{d}\left\{\|\nabla \omega\|^{2}+\left\langle T_{p}(\omega), \omega\right\rangle\right\}$.

Fact 2. $\quad \int_{\mathbf{S}^{d-1}}\left\|i_{V} d \omega\right\|^{2} d \bar{V}=c_{d}(p+1)\|d \omega\|^{2}$.

Fact 3. $\int_{\mathbf{S}^{d-1}}\left\langle i_{V} d \omega, L_{V} \omega\right\rangle d \bar{V}=c_{d}\|d \omega\|^{2}$.

Proof. Fact 1: Let $\omega \in \Lambda^{p}(M)$. We first prove that, at $x \in M$,

$$
L_{V} \omega=\nabla_{V} \omega+S_{V^{\perp}}(\omega) .
$$

In fact, recalling that $\bar{V}$ is parallel on $\mathbf{R}^{d}$ one sees that, at $x$, for all $i=1, \ldots, n$,

$$
\nabla_{e_{i}} V=S_{V^{\perp}}\left(e_{i}\right) \text {. }
$$

Hence $\left[V, e_{i}\right]=-S_{V^{\perp}}\left(e_{i}\right)$. Now it is sufficient to verify (4.31) on $p$ vectors of the frame, say $e_{i_{1}}, \ldots, e_{i_{p}}$. Then:

$$
\begin{aligned}
L_{V} \omega\left(e_{i_{1}}, \ldots, e_{i_{p}}\right) & =V \cdot \omega\left(e_{i_{1}}, \ldots, e_{i_{p}}\right)-\sum_{j=1}^{p} \omega\left(e_{i_{1}}, \ldots,\left[V, e_{i_{j}}\right], \ldots, e_{i_{p}}\right) \\
& =\nabla_{V} \omega\left(e_{i_{1}}, \ldots, e_{i_{p}}\right)+\sum_{j=1}^{p} \omega\left(e_{i_{1}}, \ldots, S_{V^{\perp}}\left(e_{i_{j}}\right), \ldots, e_{i_{p}}\right) \\
& =\nabla_{V} \omega\left(e_{i_{1}}, \ldots, e_{i_{p}}\right)+S_{V^{\perp}}(\omega)\left(e_{i_{1}}, \ldots, e_{i_{p}}\right)
\end{aligned}
$$

and (4.3) follows. We can now prove Fact 1. From (4.3):

$$
\int_{\mathbf{S}^{d-1}}\left\|L_{V} \omega\right\|^{2} d \bar{V}=\int_{\mathbf{S}^{d-1}}\left\|\nabla_{V} \omega\right\|^{2}+\int_{\mathbf{S}^{d-1}}\left\|S_{V^{\perp}}(\omega)\right\|^{2}+2 \int_{\mathbf{S}^{d-1}}\left\langle\nabla_{V} \omega, S_{V^{\perp}}(\omega)\right\rangle d \bar{V} .
$$

Now, from Lemma 4.9. part (a):

$$
\begin{aligned}
\int_{\mathbf{S}^{d-1}}\left\|\nabla_{V} \omega\right\|^{2} d \bar{V} & =\sum_{i, j=1}^{n} \int_{\mathbf{S}^{d-1}}\left\langle\bar{V}, e_{i}\right\rangle\left\langle\bar{V}, e_{j}\right\rangle d \bar{V} \cdot\left\langle\nabla_{e_{i}} \omega, \nabla_{e_{j}} \omega\right\rangle \\
& =c_{d}\|\nabla \omega\|^{2} .
\end{aligned}
$$

Let $\left(\nu_{1}, \ldots, \nu_{m}\right)$ be a local orthonormal frame in $T M^{\perp}$. Then $V^{\perp}=\sum_{\alpha=1}^{m}\left\langle\bar{V}, \nu_{\alpha}\right\rangle \nu_{\alpha}$ and

$$
\begin{aligned}
\int_{\mathbf{S}^{d-1}}\left\|S_{V^{\perp}}(\omega)\right\|^{2} d \bar{V} & =\sum_{\alpha, \beta=1}^{m} \int_{\mathbf{S}^{d-1}}\left\langle\bar{V}, \nu_{\alpha}\right\rangle\left\langle\bar{V}, \nu_{\beta}\right\rangle d \bar{V} \cdot\left\langle S_{\nu_{\alpha}}(\omega), S_{\nu_{\beta}}(\omega)\right\rangle \\
& =c_{d} \sum_{\alpha=1}^{m}\left\|S_{\nu_{\alpha}}(\omega)\right\|^{2} \\
& =c_{d}\left\langle T_{p}(\omega), \omega\right\rangle .
\end{aligned}
$$


Again from Lemma 4.9 since $V$ and $V^{\perp}$ are mutually orthogonal,

$$
\int_{\mathbf{S}^{d-1}}\left\langle\nabla_{V} \omega, S_{V^{\perp}}(\omega)\right\rangle d \bar{V}=0 .
$$

From (4.4)-4.7) one gets Fact 1.

Fact 2: Immediate from Lemma 4.9.

Fact 3: Since $V$ and $V^{\perp}$ are mutually orthogonal, one sees that

$$
\int_{\mathbf{S}^{d-1}}\left\langle i_{V} d \omega, S_{V^{\perp}}(\omega)\right\rangle d \bar{V}=0 .
$$

Hence, by (4.3),

$$
\begin{aligned}
\int_{\mathbf{S}^{d-1}}\left\langle i_{V} d \omega, L_{V} \omega\right\rangle d \bar{V} & =\int_{\mathbf{S}^{d-1}}\left\langle i_{V} d \omega, \nabla_{V} \omega\right\rangle d \bar{V} \\
& =\sum_{i, j=1}^{n} \int_{\mathbf{S}^{d-1}}\left\langle\bar{V}, e_{i}\right\rangle\left\langle\bar{V}, e_{j}\right\rangle d \bar{V} \cdot\left\langle i_{e_{i}} d \omega, \nabla_{e_{j}} \omega\right\rangle \\
& =c_{d} \sum_{i=1}^{n}\left\langle i_{e_{i}} d \omega, \nabla_{e_{i}} \omega\right\rangle \\
& =\frac{c_{d}}{p !} \sum_{i_{0}, \ldots, i_{p}=1}^{n} d \omega\left(e_{i_{0}}, \ldots, e_{i_{p}}\right) \nabla_{e_{i_{0}}} \omega\left(e_{i_{1}}, \ldots, e_{i_{p}}\right) .
\end{aligned}
$$

It remains to show that

$$
\sum_{i_{0}, \ldots, i_{p}=1}^{n} d \omega\left(e_{i_{0}}, \ldots, e_{i_{p}}\right) \nabla_{e_{i_{0}}} \omega\left(e_{i_{1}}, \ldots, e_{i_{p}}\right)=p !\|d \omega\|^{2} .
$$

Let $\Gamma$ denote the left-hand side of (4.8). Then, for all $j=0, \ldots, p$ one has, exchanging $i_{0}$ with $i_{j}$ in the arguments of $d \omega$ and renaming the indices,

$$
\Gamma=(-1)^{j} \sum_{i_{0}, \ldots, i_{p}=1}^{n} d \omega\left(e_{i_{0}}, \ldots, e_{i_{p}}\right) \nabla_{e_{i_{j}}} \omega\left(e_{i_{0}}, \ldots, \hat{e}_{i_{j}}, \ldots, e_{i_{p}}\right),
$$

so that

$$
\begin{aligned}
(p+1) \Gamma & =\sum_{i_{0}, \ldots, i_{p}=1}^{n} d \omega\left(e_{i_{0}}, \ldots, e_{i_{p}}\right)\left(\sum_{j=0}^{p}(-1)^{j} \nabla_{e_{i_{j}}} \omega\left(e_{i_{0}}, \ldots, \hat{e}_{i_{j}}, \ldots, e_{i_{p}}\right)\right) \\
& =\sum_{i_{0}, \ldots, i_{p}=1}^{n}\left(d \omega\left(e_{i_{0}}, \ldots, e_{i_{p}}\right)\right)^{2} \\
& =(p+1) !\|d \omega\|^{2}
\end{aligned}
$$

and (4.8) follows.

It remains to prove the assertion on sharpness in Theorem 2.1. If $M=\mathbf{S}^{n} \rightarrow$ $\mathbf{R}^{n+1}$ is the standard immersion, and if $\omega$ is an eigenform associated to $\mu_{1, p}^{\prime \prime}\left(\mathbf{S}^{n}\right)$, then we must have equality in Theorem 2.1 simply because we have equality in Theorem 2.3 (which is a consequence of Theorem 2.1, see its proof below).

One can also prove directly that, for all fields $V$ considered in the proof, $i_{V} \omega$ is an eigenform associated to $\mu_{1, p}^{\prime \prime}\left(\mathbf{S}^{n}\right)-n+2 p$, which coincides with $\mu_{1, p-1}^{\prime \prime}\left(\mathbf{S}^{n}\right)$ by 
(4.9) below. Hence, in the min-max principle of Lemma 4.7 one has the equality for all $V$, and this implies the equality in the final formula.

Next, we prove a useful lemma. Let $M$ be any Riemannian manifold with boundary, and let $\tilde{S}$ be the shape operator of the immersion of $\partial M$ into $M$, relative to the inner unit normal $\tilde{\nu}$ of $\partial M$ (hence $\tilde{S}(X)=-\nabla_{X} \tilde{\nu}$ for all $X \in T(\partial M)$ ). By derivation, we extend $\tilde{S}$ to a field of endomorphisms of $\Lambda^{p}(\partial M), p=1, \ldots, n-1$, denoted by $\tilde{S}_{p}$. Note that the eigenvalues of $\tilde{S}_{p}$ are the $p$-curvatures of $\partial M$; hence all these eigenvalues are bounded below by $\sigma_{p}$.

Lemma 4.10. (a) Let $M$ be any Riemannian manifold with boundary, and assume that $\omega \in \Lambda^{p}(M)$ satisfies the absolute conditions on $\partial M$. If $\tilde{\nu}$ is the inner unit normal to $\partial M$, then, at any point of the boundary,

$$
\left\langle\nabla_{\tilde{\nu}} \omega, \omega\right\rangle=\left\langle\tilde{S}_{p}\left(J^{*} \omega\right), J^{*} \omega\right\rangle,
$$

where $J^{*} \omega$ is the restriction of $\omega$ to $\partial M$.

(b) Let $\sigma_{p}$ be a lower bound of the p-curvatures of $\partial M$. Then

$$
\int_{M} \Delta\left(\|\omega\|^{2}\right) \geq 2 \sigma_{p} \int_{\partial M}\|\omega\|^{2} .
$$

Proof. (a) Take a local orthonormal frame $\left(e_{1}, \ldots, e_{n-1}\right)$ of $T(\partial M)$ which is geodesic at the given point $y \in \partial M$. Since $i_{\tilde{\nu}} \omega=0$, one has, at $y$ :

$$
\left\langle\nabla_{\tilde{\nu}} \omega, \omega\right\rangle=\sum_{i_{1}<\cdots<i_{p}} \nabla_{\tilde{\nu}} \omega\left(e_{i_{1}}, \ldots, e_{i_{p}}\right) \omega\left(e_{i_{1}}, \ldots, e_{i_{p}}\right) .
$$

Expliciting the condition $i_{\tilde{\nu}} d \omega\left(e_{i_{1}}, \ldots, e_{i_{p}}\right)=0$ at $y$, we get

$$
\begin{aligned}
\nabla_{\tilde{\nu}} \omega\left(e_{i_{1}}, \ldots, e_{i_{p}}\right) & =-\sum_{j=1}^{p}(-1)^{j} \nabla_{e_{i_{j}}} \omega\left(\tilde{\nu}, e_{i_{1}}, \ldots, \hat{e}_{i_{j}}, \ldots, e_{i_{p}}\right) \\
& =-\sum_{j=1}^{p}(-1)^{j} \omega\left(\nabla_{e_{i_{j}}} \tilde{\nu}, e_{i_{1}}, \ldots, \hat{e}_{i_{j}}, \ldots, e_{i_{p}}\right) \\
& =\sum_{j=1}^{p}(-1)^{j} \omega\left(\tilde{S}\left(e_{i_{j}}\right), e_{i_{1}}, \ldots, \hat{e}_{i_{j}}, \ldots, e_{i_{p}}\right) \\
& =\tilde{S}_{p}\left(J^{*} \omega\right)\left(e_{i_{1}}, \ldots, e_{i_{p}}\right)
\end{aligned}
$$

and (a) follows. As the eigenvalues of $\tilde{S}_{p}$ are bounded below by $\sigma_{p}$, one has on $\partial M$ :

$$
\frac{\partial}{\partial \widetilde{\nu}}\|\omega\|^{2}=2\left\langle\nabla_{\tilde{\nu}} \omega, \omega\right\rangle \geq 2 \sigma_{p}\left\|J^{*} \omega\right\|^{2}=2 \sigma_{p}\|\omega\|^{2}
$$

because $i_{\tilde{\nu}} \omega=0$. Part (b) now follows from Green's formula.

Now, we write explicitly the first Hodge eigenvalues of spheres and hemispheres. By the calculation in [5],

$$
\left\{\begin{array}{l}
\mu_{1, p}^{\prime}\left(\mathbf{S}^{n}\right)=p(n-p+1) \quad \text { for } \quad p=1, \ldots, n, \\
\mu_{1, p}^{\prime \prime}\left(\mathbf{S}^{n}\right)=(p+1)(n-p) \text { for } \quad p=0, \ldots, n-1,
\end{array}\right.
$$

hence, for $p=1, \ldots, n-1$,

$$
\mu_{1, p}^{\prime \prime}\left(\mathbf{S}^{n}\right)-\mu_{1, p}^{\prime}\left(\mathbf{S}^{n}\right)=n-2 p
$$


and

$$
\mu_{1, p}\left(\mathbf{S}^{n}\right)=\left\{\begin{array}{l}
n \quad \text { if } p=0, n \\
p(n-p+1) \quad \text { if } \quad 1 \leq p \leq[n / 2] \\
(p+1)(n-p) \text { if } 1+[n / 2] \leq p \leq n-1 .
\end{array}\right.
$$

Now the above equalities continue to hold if $\mathbf{S}^{n}$ is replaced by a hemisphere $\mathbf{S}_{+}^{n}$. In fact, identify the hemisphere with $\mathbf{S}_{+}^{n}=\left\{\left(x_{1}, \ldots, x_{n+1}\right) \in \mathbf{S}^{n}: x_{n+1} \geq 0\right\}$ and let $\sigma: \mathbf{S}^{n} \rightarrow \mathbf{S}^{n}$ denote the reflection $\sigma\left(x_{1}, \ldots, x_{n}, x_{n+1}\right)=\left(x_{1}, \ldots, x_{n},-x_{n+1}\right)$. As the Laplacian commutes with $\sigma$, the restriction to $\mathbf{S}_{+}^{n}$ of any eigenform $\omega$ associated to $\mu_{1, p}^{\prime}\left(\mathbf{S}^{n}\right)$ (resp. $\mu_{1, p}^{\prime \prime}\left(\mathbf{S}^{n}\right)$ ) which is invariant under $\sigma$ and nonzero (there exist such) will be an eigenform on $\mathbf{S}_{+}^{n}$ associated to the same eigenvalue and satisfying the absolute boundary conditions. Now an obvious reflection argument shows that the spectrum of the Laplacian on $p$-forms on $\mathbf{S}_{+}^{n}$, for the absolute (or relative) conditions is a subset of the corresponding $p$-spectrum of $\mathbf{S}^{n}$, hence the assertion.

Proof of Theorem 2.3. Let $M^{n} \rightarrow \mathbf{R}^{d}$ be an isometric immersion, such that $M$ is either closed or has a $p$-convex boundary. We have to show that, for all $1 \leq p \leq$ $n-1$,

$$
\mu_{1, p}^{\prime \prime}(M)-\mu_{1, p}^{\prime}(M) \geq \frac{1}{p} \inf _{M}\left(W_{p}-T_{p}\right) .
$$

Let $\omega$ be an eigenform associated to $\mu_{1, p}^{\prime \prime}(M)$ having unit $L^{2}$-norm. By the Bochner formula and Lemma 4.10 one has:

$$
\begin{aligned}
\mu_{1, p}^{\prime \prime}(M) & =\int_{M}\left\{\|\nabla \omega\|^{2}+\frac{1}{2} \Delta\left(\|\omega\|^{2}\right)+\left\langle W_{p}(\omega), \omega\right\rangle\right\} \\
& \geq \int_{M}\left\{\|\nabla \omega\|^{2}+\left\langle W_{p}(\omega), \omega\right\rangle\right\}+\sigma_{p} \int_{\partial M}\|\omega\|^{2} .
\end{aligned}
$$

Now $\int_{M}\|d \omega\|^{2}=\mu_{1, p}^{\prime \prime}(M)$, and then, by Theorem 2.1 applied to $\omega$,

$$
\int_{M}\|\nabla \omega\|^{2} \geq p \mu_{1, p}^{\prime}(M)-(p-1) \mu_{1, p}^{\prime \prime}(M)-\int_{M}\left\langle T_{p}(\omega), \omega\right\rangle .
$$

Inserting in (4.12), we get:

$$
p\left(\mu_{1, p}^{\prime \prime}(M)-\mu_{1, p}^{\prime}(M)\right) \geq \int_{M}\left\langle\left(W_{p}-T_{p}\right)(\omega), \omega\right\rangle+\sigma_{p} \int_{\partial M}\|\omega\|^{2} .
$$

As $\partial M$ is $p$-convex one has $\sigma_{p} \geq 0$ and the assertion follows.

If $M=\mathbf{S}^{n}$, isometrically immersed in $\mathbf{R}^{n+1}$, then $W_{p}=p(n-p) \cdot I d$ and $T_{p}=p^{2} \cdot I d$, hence

$$
\frac{1}{p}\left(W_{p}-T_{p}\right)=(n-2 p) \cdot I d
$$

at all points. The equality now follows from the calculation in (4.10).

Proof of Theorem 2.8. Let $M$ be a convex domain of $\mathbf{S}^{n}$, and $\mathbf{S}_{+}^{n}$ the hemisphere.

Let $G_{p}=\mu_{1, p}(M)-\mu_{1, p-1}(M)$, and:

$$
\bar{G}_{p}:=\mu_{1, p}\left(\mathbf{S}_{+}^{n}\right)-\mu_{1, p-1}\left(\mathbf{S}_{+}^{n}\right)=\left\{\begin{array}{l}
0 \quad \text { if } p=1, n ; \\
n-2 p+2 \text { if } 2 \leq p \leq[n / 2] ; \\
2 n-4 p+2 \quad \text { if } p=[n / 2]+1 ; \\
n-2 p \text { if }[n / 2]+2 \leq p \leq n-1
\end{array}\right.
$$


(see (4.11)). Part (a) says that, for all $p=1, \ldots, n$,

$$
G_{p} \geq \bar{G}_{p} .
$$

We prove this by direct comparison with (4.13). Now $G_{n} \geq 0$ is always true. Let $p=1, \ldots, n-1$. By Theorem 2.3

$$
\mu_{1, p}^{\prime \prime}-\mu_{1, p}^{\prime} \geq n-2 p .
$$

As $\mu_{1,1}^{\prime \prime} \geq \mu_{1,1}^{\prime}$, one has $G_{1} \geq 0=\bar{G}_{1}$ and (4.14) is true for $p=1$. Then, let $p=2, \ldots, n-1$. Recall that $\mu_{1, p}=\min \left\{\mu_{1, p}^{\prime}, \mu_{1, p}^{\prime \prime}\right\}$. We will prove below that:

$$
\left\{\begin{array}{l}
\text { If } \mu_{1, p}=\mu_{1, p}^{\prime}, \text { then } G_{p} \geq n-2 p+2 . \\
\text { If } \mu_{1, p}=\mu_{1, p}^{\prime \prime}, \text { then } G_{p} \geq \max \{n-2 p, 2 n-4 p+2\} .
\end{array}\right.
$$

Given (4.16), one proceeds as follows. If $2 \leq p \leq[n / 2]$ then $\mu_{1, p}^{\prime \prime}-\mu_{1, p}^{\prime} \geq n-2 p \geq 0$; hence we are in the first case of (4.16) and $G_{p} \geq \bar{G}_{p}$. If $p \geq 1+[n / 2]$, just observe that $n-2 p+2 \geq \max \{n-2 p, 2 n-4 p+2\}$ and then $G_{p} \geq \bar{G}_{p}$ in both cases of (4.16).

It remains to show (4.16). It follows by a repeated use of (4.15) and Hodge decomposition. Assume that $\mu_{1, p}=\mu_{1, p}^{\prime}$; then

$$
\mu_{1, p}=\mu_{1, p}^{\prime}=\mu_{1, p-1}^{\prime \prime} \geq \mu_{1, p-1}^{\prime}+n-2 p+2 \geq \mu_{1, p-1}+n-2 p+2,
$$

and the first part follows. Now assume that $\mu_{1, p}=\mu_{1, p}^{\prime \prime}$. Then:

$$
\mu_{1, p}=\mu_{1, p}^{\prime \prime} \geq \mu_{1, p}^{\prime}+n-2 p=\mu_{1, p-1}^{\prime \prime}+n-2 p ;
$$

as $\mu_{1, p-1}^{\prime \prime} \geq \mu_{1, p-1}$, one certainly has $\mu_{1, p}-\mu_{1, p-1} \geq n-2 p$. But one also has $\mu_{1, p-1}^{\prime \prime} \geq \mu_{1, p-1}^{\prime}+n-2 p+2 \geq \mu_{1, p-1}+n-2 p+2$; substituting in (4.17), one gets $\mu_{1, p}-\mu_{1, p-1} \geq 2 n-4 p+2$ and (4.16) is proved.

Proof of (b). We show that for all $p=0, \ldots,[n / 2]$ one has $\lambda_{1, p}(M) \geq \mu_{1, p}(M)$. By the Hodge duality $\lambda_{1, p}=\mu_{1, n-p}$. If $p \leq[n / 2]$, then $n-p \geq p$ and then:

$$
\mu_{1, n-p}-\mu_{1, p} \geq \mu_{1, n-p}\left(\mathbf{S}_{+}^{n}\right)-\mu_{1, p}\left(\mathbf{S}_{+}^{n}\right)=0 .
$$

Finally the formula

$$
\begin{aligned}
\mu_{1, p}(M)-\mu_{1,0}(M) \geq & \mu_{1, p}\left(\mathbf{S}_{+}^{n}\right)-\mu_{1,0}\left(\mathbf{S}_{+}^{n}\right) \\
& =\left\{\begin{array}{l}
0 \text { if } p=1, n ; \\
(p-1)(n-p) \text { if } 2 \leq p \leq[n / 2] \\
p(n-p-1) \text { if } 1+[n / 2] \leq p \leq n-1 .
\end{array}\right.
\end{aligned}
$$

follows directly from part (a) and the calculation (4.11).

\subsection{Proof of Theorem 3.1 .}

Definition 4.11. Let $(n-1) K$ be a lower bound of the Ricci curvature of $M$ and $H$ a lower bound of the mean curvature of $\partial M$. Define the constant $A=A(K, H, R)$, depending on $K$ and $H$ and the inner radius $R$, by:

$$
A=\inf \left\{-\frac{\theta_{K, H}^{\prime}(r)}{\theta_{K, H}(r)}, 0 \leq r<R\right\},
$$


where $\theta_{K, H}(r)=\left[s_{K}^{\prime}(r)-H s_{K}(r)\right]^{n-1}$ and

$$
s_{K}(r)=\left\{\begin{array}{l}
\frac{1}{\sqrt{K}} \sin (r \sqrt{K}) \quad \text { if } \quad K>0 ; \\
r \quad \text { if } \quad K=0 ; \\
\frac{1}{\sqrt{|K|}} \sinh (r \sqrt{|K|}) \quad \text { if } \quad K<0 .
\end{array}\right.
$$

Remark 4.12. Note that, if $K \geq 0$, or $K<0$ and $H \geq \sqrt{|K|}$, then $A=(n-1) H$.

In fact, under any of the above two conditions the function $-\frac{\theta_{K, H}^{\prime}(r)}{\theta_{K, H}(r)}$ is easily seen to be nondecreasing in $r$. The constant $A$ comes out of a Heintze-Karcher type estimate, needed in the proof of Theorem 3.1

Let us prove the theorem. Let $M$ be a Riemannian manifold with smooth boundary, and assume that the nonnegative function $\phi$ satisfies $\Delta \phi \leq \mu \phi$ on $M$, for some $\mu \in \mathbf{R}$. Fix any $t \in\left(0, \frac{\pi}{2 R}\right)$. We must prove that, if $\mu \leq \frac{A^{2}}{4}+t^{2}$, then

$$
\int_{\partial M} \phi \geq\left[\frac{A}{2}+t \cot (R t)\right] \cdot \int_{M} \phi .
$$

We use distance function methods. Let $\rho(x)=\operatorname{dist}(x, \partial M)$ be the distance function from the boundary of $M$. Given $\phi \in C^{\infty}(M)$, we consider the function of $r \in[0, \infty)$ defined by

$$
F(r)=\int_{M(r)} \phi
$$

where $M(r)=\{x \in M: \rho(x)>r\}$ is the set of all points whose distance from the boundary is greater than $r$. As $\rho$ is only Lipschitz on $M, F(r)$ will be only Lipschitz on the half-line (we refer to [15] for full technical details about the functions $\rho$ and $F$ ). Nevertheless, it will be useful to examine the distributional derivatives of $F(r)$; this will lead to the following lemma.

Lemma 4.13. Assume that $\phi$ is nonnegative and satisfies $\Delta \phi \leq \mu \phi$ on $M$ for some $\mu \in \mathbf{R}$. Then the function $F(r)$ satisfies the following differential inequality, in the sense of distributions on the half-line:

$$
F^{\prime \prime}+A F^{\prime}+\mu F \geq 0
$$

Note. All distributions considered in the proof are of order zero, i.e., are Radon measures.

Proof of Lemma 4.13. The distance function $\rho$ is Lipschitz on $M$, and smooth on the set $\tilde{M}=M \backslash C u t(\partial M)$, where $C u t(\partial M)$ denotes the cut-locus of the normal exponential map of $\partial M(C u t(\partial M)$ has measure zero in $M)$. One has, a.e. on $[0, \infty)$

$$
F^{\prime}(r)=-\int_{\rho^{-1}(r) \cap \tilde{M}} \phi,
$$

the measure on the smooth hypersurface $\rho^{-1}(r) \cap \tilde{M}$ being the one induced by the Riemannian metric of $M$. Let $\Delta \rho$ be the distributional Laplacian of $\rho$. From the mean-value lemma (see [15], formula (7), p. 517) the second derivative of $F$ is given by

$$
F^{\prime \prime}=-\int_{M(r)} \Delta \phi+\rho_{*}(\phi \Delta \rho),
$$


where $\rho_{*}(\phi \Delta \rho)$ denotes the distribution on $[0, \infty)$ given by the push-forward of $\phi \Delta \rho$ by $\rho$. The lemma will follow from the inequality

$$
\rho_{*}(\phi \Delta \rho) \geq-A F^{\prime},
$$

which we now proceed to show. One has the splitting

$$
\Delta \rho=\Delta_{\text {reg }} \rho+\Delta_{\text {cut }} \rho,
$$

where the regular part $\Delta_{\text {reg }} \rho$ is the Laplacian of the restriction of $\rho$ to $\tilde{M}$, and the singular part $\Delta_{c u t} \rho$ is a positive distribution supported on the cut-locus (see 15], Lemma 2.1). Fix a point $x \in \tilde{M}$ and let $(r, y) \in[0, \infty) \times \partial M$ be its normal coordinates: that is, $r=\rho(x)$ and $y$ is the foot of the (unique) minimizing geodesic joining $x$ and $\partial M$. In these coordinates

$$
\Delta_{r e g} \rho(r, y)=-\frac{1}{\Theta} \frac{\partial \Theta}{\partial r}(r, y)
$$

where $\Theta(r, y)$ denotes the density of the Riemannian volume form. By the HeintzeKarcher estimates, one has then

$$
-\frac{1}{\Theta} \frac{\partial \Theta}{\partial r}(r, y) \geq-\frac{\theta_{K, H}^{\prime}}{\theta_{K, H}}(r) \geq A
$$

which, together with the positiveness of $\Delta_{c u t} \rho$, implies

$$
\Delta \rho \geq A \text {. }
$$

Finally, as $\phi$ is nonnegative on $M$,

$$
\rho_{*}(\phi \Delta \rho) \geq A \rho_{*}(\phi)=-A F^{\prime},
$$

the last equality following from the co-area formula and the definition of pushforward, which imply

$$
\rho_{*}(\phi)(r)=\int_{\rho^{-1}(r) \cap \tilde{M}} \phi=-F^{\prime}(r)
$$

for almost all $r$.

Proof of Theorem 3.1 (continued). Assume that $\mu \leq \frac{A^{2}}{4}+t^{2}:=\mu_{t}$. Then $\Delta \phi \leq$ $\mu_{t} \phi$ and, by Lemma 4.13 the function $F(r)=\int_{M(r)} \phi$ will satisfy the differential inequality

$$
F^{\prime \prime}+A F^{\prime}+\mu_{t} F \geq 0
$$

Let $y(r)$ be the solution of the corresponding differential equation, having the same initial conditions of $F$ :

The explicit solution is

$$
\left\{\begin{array}{l}
y^{\prime \prime}+A y^{\prime}+\mu_{t} y=0, \\
y(0)=F(0)=\int_{M} \phi, \\
y^{\prime}(0)=F^{\prime}(0)=-\int_{\partial M} \phi .
\end{array}\right.
$$

$$
y(r)=e^{-\frac{A r}{2}}\left(d_{1} \cos t r-d_{2} \sin t r\right),
$$

where

$$
d_{1}=\int_{M} \phi \quad \text { and } \quad d_{2}=\frac{1}{t}\left[\int_{\partial M} \phi-\frac{A}{2} \int_{M} \phi\right]
$$


Now, even if Lemma 4.13 holds only in the sense of distributions, the usual comparison arguments still hold, and we conclude that $F(r) \geq y(r)$ for $r \in\left[0, R_{t}\right]$, where $R_{t}$ is the first zero of $y(r)$ (note that $R_{t}>0$ because $y(0)>0$ ). In particular, as $R=\max _{M} \rho$, one has $F(R)=0$ and $R \geq R_{t}$. Since $t R_{t} \leq t R \in(0, \pi / 2)$, and $\cot \left(t R_{t}\right)=\frac{d_{2}}{d_{1}}$, one gets

which is the final statement.

$$
\cot (t R) \leq \frac{d_{2}}{d_{1}}
$$

\section{REFERENCES}

1. I. Chavel, Eigenvalues in Riemannian geometry, Academic Press, 1984. MR 86g:58140

2. B. Colbois and G. Courtois, A note on the first eigenvalue of the Laplacian acting on p-forms, Manuscripta Math. 68 (1990), 143-160. MR 91g:58290

3. J. Dodziuk, Eigenvalues of the Laplacian on forms, Proc Amer. Math. Society 85, 3 (1982). MR 84k:58223

4. L. Friedlander, Some inequalities between Dirichlet and Neumann eigenvalues, J. Arch. Ration. Mech. Anal. 116, No.2, (1991), 153-160. MR 93h:35146

5. S. Gallot and D. Meyer, Opérateur de courbure et Laplacien des formes différentielles d'une variété riemannienne, J. Math. Pures Appl. 54 (1975), 259-284. MR 56:13128

6. S. Gallot and D. Meyer, D'un résultat hilbertien à un principe de comparaison entre spectres. Applications, Ann. Scient. Ec. Norm. Sup., 4 Serie, 21 (1988), 561-591. MR 90k:58236

7. G. Gentile and V. Pagliara, Riemannian metrics with large first eigenvalue on forms of degree p, Proc. Amer. Math. Soc. 123 (1995), no. 12, 3855-3858. MR 96b:58115

8. P. Guerini, Spectre du Laplacien de Hodge-de Rham: Estimées sur les Variétés Convexes, Preprint.

9. P. Guerini, Prescription du Spectre du Laplacien de Hodge-de Rham, Preprint Universität Zürich 2002.

10. P. Li and S-T. Yau, Estimates of eigenvalues of a compact Riemannian manifold, Proc. Symp. Pure Math. 36 (1980), 205-239. MR 81i:58050

11. J.K. McGowan, The p-spectrum of the Laplacian on compact hyperbolic three manifolds, Math. Ann. 297 (1993), 4, 725-745. MR 94g:58239

12. H.P. McKean, An upper bound for the spectrum of $\Delta$ on a manifold of negative curvature, J. Diff. Geom. 4 (1970), 359-366. MR 42:1009

13. L. Payne and H. Weinberger, Lower bounds for vibration frequencies of elastically supportes membranes and plates, J. Soc. Ind. Appl. Math. 5 (1957), 171-182. MR 19:1110c

14. R.C. Reilly, On the first eigenvalue of the Laplacian for compact submanifolds of Euclidean space, Comment. Math. Helv. 52 (1977), no. 4, 525-533. MR 58:2657

15. A. Savo, A mean-value lemma and applications, Bull. Soc. Math. France 129 (2001), no. 4, 505-542.

16. J. Takahashi, On the gap between the first eigenvalues of the Laplacian on functions and 1-forms, J. Math. Soc. Japan 53 (2001), no. 2, 307-320. MR 2002a:58031

17. J. Takahashi, On the gap between the first eigenvalues of the Laplacian on functions and 1-forms, Ann. Glob. Anal. Geom. to appear.

18. H.F. Weinberger, An isoperimetric inequality for the n-dimensional free membrane problem, J. Rational Mech. Anal. 5 (1956), 633-636. MR 18:63c

Institut für Mathematik, Universität Zürich Irchel, Winterthurerstrasse 90, CH8057 ZürICH, SwITZERLAND

E-mail address: pguerini@math.unizh.ch

Dipartimento di Metodi e Modelli Matematici, Università di Roma I la Sapienza, Via Antonio Scarpa 16, 00161 Roma, Italy

E-mail address: savo@dmmm.uniroma1.it 